TI 2017-010/VI

Tinbergen Institute Discussion Paper
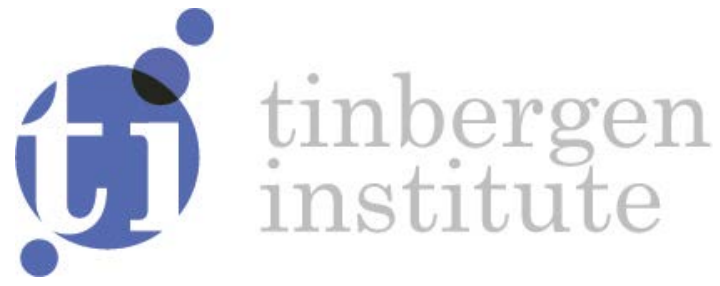

\title{
Welfare Effects of Short-Time Compensation
}

Helge Braun ${ }^{1}$

Björn Brügemann²

${ }^{1}$ Ruhr Graduate School in Economics

${ }^{2}$ VU Amsterdam, Tinbergen Institute, CESifo, and IZA 
Tinbergen Institute is the graduate school and research institute in economics of Erasmus University Rotterdam, the University of Amsterdam and VU University Amsterdam.

Contact: discussionpapers@tinbergen.nl

More TI discussion papers can be downloaded at http://www.tinbergen.nl

Tinbergen Institute has two locations:

Tinbergen Institute Amsterdam

Gustav Mahlerplein 117

1082 MS Amsterdam

The Netherlands

Tel.: $+31(0) 205984580$

Tinbergen Institute Rotterdam

Burg. Oudlaan 50

3062 PA Rotterdam

The Netherlands

Tel.: +31(0)104088900 


\title{
Welfare Effects of Short-Time Compensation*
}

\author{
Helge Braun $\quad$ Björn Brügemann ${ }^{\mathrm{b}, \mathrm{c}}$
}

January 2017

\begin{abstract}
We study welfare effects of public short-time compensation (STC) in a model in which firms respond to idiosyncratic profitability shocks by adjusting employment and hours per worker. Introducing STC substantially improves welfare by mitigating distortions caused by public unemployment insurance (UI), but only if firms have access to private insurance. Otherwise firms respond to low profitability by combining layoffs with long hours for remaining workers, rather than by taking up STC. Optimal STC is substantially less generous than UI even when firms have access to private insurance, and equally generous STC is worse than not offering STC at all.
\end{abstract}

Keywords: Short-Time Compensation, Unemployment Insurance, Welfare

JEL Classification: J65

\footnotetext{
${ }^{*}$ We are grateful to Klaudia Michalek for her participation in early stages of the project. We would like to thank Pierre Cahuc, Rob Euwals, Moritz Kuhn, and numerous seminar and conference participants for helpful comments and

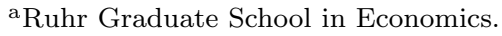

${ }^{\mathrm{b}}$ VU Amsterdam, Tinbergen Institute, CESifo, and IZA.

${ }^{\mathrm{c} C}$ Corresponding author at: VU Amsterdam, Department of Economics, De Boelelaan 1105, 1081 HV Amsterdam, Netherlands.

E-mail addresses: b.a.brugemann@vu.nl (B. Brügemann), helge.braun@rgs-econ.de (H. Braun).
} discussions. 


\section{Introduction}

Virtually all developed countries have public unemployment insurance (UI) systems. In addition, many countries run public short-time compensation (STC) schemes, which pay benefits to workers that have not lost their job but are working reduced hours. In contrast to UI, STC has not been a universal component of social insurance systems in developed countries. Before the 2008-2009 crisis, STC schemes existed in 18 out of 33 OECD countries. Such schemes increased in popularity during the crisis, with many countries expanding existing schemes and others introducing new schemes on a temporary basis. ${ }^{1}$

This increase in the popularity of STC has also revived academic interest in this policy instrument. Recent research has primarily focussed on employment effects of STC during the crisis. ${ }^{2}$ What has received little attention, both in recent and earlier work, are effects of STC on social welfare. This contrasts with UI, which has been studied extensively from a welfare perspective. In this paper we study welfare effects of STC in a setting in which UI is socially optimal, consistent with the observation that UI is a universal feature of social insurance systems in developed countries. We ask if introducing STC can improve welfare in a situation in which the instrument of UI is already used optimally.

We study this question in a static model of implicit contracts, building on existing theoretical work on STC. Workers are risk averse and ex ante heterogeneous in that they are either attached to a firm or unattached. Both attached and unattached workers can be unemployed ex post. We follow existing work in not separating the role of workers and employers: workers attached to a firm are both suppliers of its labor input as well as its owners. Firms are subject to idiosyncratic profitability shocks, and can adjust through a combination of layoffs and work sharing in the sense of adjusting hours per worker. Profitability shocks are interpreted as temporary, and layoffs are interpreted as temporary layoffs that do not break attachment to the firm. The government has two policy instruments, UI and STC. UI is a payment to each unemployed worker, where a worker is considered unemployed if working zero hours. ${ }^{3}$ Thus workers are unemployed either because they are unattached or on temporary layoff. UI is the only source of income for unattached workers. STC is a payment for each hour by which working time is reduced below some threshold of normal hours. We allow for the possibility that eligibility for STC may require a minimum reduction in hours per worker, a common feature of existing STC schemes. ${ }^{4}$ The

\footnotetext{
${ }^{1}$ Arpaia et al. (2010) and Hijzen and Venn (2011) survey STC schemes.

2 See for example Arpaia et al. (2010), Hijzen and Venn (2011), Boeri and Bruecker (2011), Cahuc and Carcillo (2011), Hijzen and Martin (2013), and Balleer et al. (2016).

${ }^{3}$ Unemployment here means eligibility for benefits rather than search activity. Unattached workers are eligible. For consistency with the typical UI system, their status should be interpreted as including having worked in the recent past.

${ }^{4}$ In their survey of STC schemes in the OECD, Hijzen and Venn (2011) find that 15 out of 24 countries have minimum
} 
government balances the budget through a linear tax on total hours.

When studying public insurance, it is important to take into account agents' access to private insurance (PI). The premise of implicit contract models is that firms are an important source of PI, due to firms' superior access to financial markets relative to workers. Firms are heterogeneous in the extent of this access, however, and we are interested in how the extent of access to PI affects the response of firms to the availability of STC, and in turn the welfare effects of STC. For simplicity, we restrict attention to two polar scenarios: either firms have access to perfect PI, or they have no access to PI. ${ }^{5}$

Welfare effects of UI in this setting are well understood. It has a positive effect on utilitarian welfare via redistribution towards unattached workers. If firms lack access to perfect PI, UI also provides insurance to attached workers. The cost of UI is a distortion of labor inputs, as firms do not internalize the impact of layoffs on the government budget (Feldstein, 1976).

Starting from a situation in which the level of UI is chosen to maximize social welfare, the introduction of STC can affect welfare through two channels. First, since private labor input decisions are distorted by UI, STC affects welfare through its impact on these decisions. This is the only welfare effect of STC when firms have access to perfect PI. If firms lack such access, STC also has a direct insurance effect, since it reallocates resources across firms with different realizations of profitability.

Our analysis proceeds in two main steps. First, we analyze firms' decisions for given values of the policy instruments. In particular, we characterize how firms adjust labor inputs in response to profitability shocks, conditional on the decision to take up STC. This is well known for the case of perfect PI: when profitability is sufficiently low for layoffs to be optimal, a further drop in profitability causes lower employment, while hours per worker remain constant. For the case of no PI and for our specification of preferences, which is a standard specification in macroeconomics, we establish a new comparative statics property: the availability of UI induces firms to respond to a drop in profitability by increasing hours per worker. This occurs because lower profitability raises the marginal utility of consumption relative to the marginal disutility of working longer hours for workers with positive hours. ${ }^{6}$

hours reductions. They range from $4 \%$ to $40 \%$, with an average around $20 \%$.

${ }^{5}$ In implicit contract models that separate the roles of workers and employers, the extent of a firm's access to PI is usually captured indirectly via the risk aversion of the employer, with risk neutrality at one end of the spectrum. In contrast, we model access to PI directly.

${ }^{6}$ This property continues to apply if the roles of workers and employers are separated. If employers are effectively risk neural, either because of preferences or access to PI, then hours are constant across profitability levels for which layoffs are optimal, exactly as in our case of perfect PI. As soon as employers are effectively risk averse, workers' marginal utility of consumption relative to their marginal utility of working longer hours is higher at lower levels of profitability, inducing higher optimal hours when profitability is low. 
This property turns out to be key in shaping the welfare effects of STC when firms lack access to PI.

In the second step, we study welfare-maximizing choices of UI and STC. We rely on computational experiments, calibrating the model by targeting features of the US labor market. We obtain two main results. First, introducing STC substantially improves welfare, but only if firms have access to PI. If firms have such access, STC can mitigate excessive layoffs caused by UI. This mechanism fails if firms lack access to PI, due to the comparative statics property discussed above. In the absence of STC, unprofitable firms would choose layoffs combined with high hours per worker, and this makes the take-up of STC unappealing. Instead, STC is taken up by firms with intermediate profitability, and for most of these firms STC merely distorts hours. This pattern of take-up also implies that STC has a direct negative insurance effect, but quantitatively this is relatively unimportant. Overall, adopting the same level that is optimal in the case of PI leads to a moderate welfare loss. Thus our model suggests that to the extent that the government can observe firms' access to PI, it is desirable to have different levels of STC for different groups of firms. Our second main result is that optimal STC is substantially less generous than UI even when firms have access to PI. In our model there is no reason to expect that equal generosity is optimal, since the optimal levels of STC and UI are governed by different trade-offs: as discussed above, optimal UI is governed by the trade-off between the benefits of redistribution and insurance and the cost of inducing excessive layoffs, while optimal STC balances the benefit of reducing these excessive layoffs against the cost of distorting hours in firms that would abstain from layoffs even in the absence of STC. According to our computational experiments, STC should be about one third as generous as UI. Furthermore, equally generous STC is worse than not offering STC at all. This is important, given that equal generosity of STC and UI is a common feature of existing schemes. ${ }^{7}$

We contribute both to the literature using implicit contract models to study STC, and the broader literature using such models to study the response of layoffs and hours per worker to shocks. Our analysis of STC builds heavily on Burdett and Wright (1989, henceforth BW) and Wright and Hotchkiss (1988, henceforth WH). BW use an implicit contract model to study effects of UI and STC on layoffs, hours per worker, and wages. A key feature of their model is that laissez faire is socially optimal. Their analysis is focussed on the distortions induced by UI and STC. They find that while UI distorts the level of employment, STC distorts hours per worker. WH extend the analysis of BW in several directions, two of which are important for our purposes. While BW consider a model in which workers and employers are distinct agents, WH also consider a simplified model which abstracts from this heterogeneity. We

\footnotetext{
${ }^{7}$ More precisely, it is common for replacement rates received by workers to be equal for UI and STC. What matters in our model is the generosity from the joint perspective of workers and employers. Some programs such as Germany's are effectively less generous than UI by imposing additional costs of utilizing STC on employers.
} 
adopt this simplification. Second, WH use this simplified model to analyze social welfare. As in BW, having neither UI nor STC is socially optimal. Alternatively, UI and STC can be neutralized through full experience rating. If there is no UI in their model, then it is also optimal to have no STC. Neither BW nor WH address the question whether a positive level of STC would be optimal given that the level of UI is positive. Addressing this question is important, given that UI is universal across developed economies. Our main contribution to this literature is to fill this gap. We generate a reason for the existence of UI through the presence of unattached workers. ${ }^{8}$ In turn, the existence of UI gives rise to a nontrivial trade-off for STC, since STC can mitigate distortions induced by UI.

Since the work of BW and $\mathrm{WH}$ on STC, there has been tremendous progress in the development of dynamic models of the labor market. Nonetheless, static implicit contract models remain a natural starting point for studying the welfare effects of STC because they combine the following three features: (i) specificity of employment relationships, captured by the attachment of workers to firms, (ii) multi-worker firms, adjusting at both the extensive and the intensive margin, (iii) private insurance arrangements among the agents attached to a firm, in a setting with incomplete markets. While there are dynamic models capturing these features individually, tractable models capturing them jointly have not yet been developed. Of course, a static model does not allow us to evaluate some potential effects of STC, such as the concern that STC reduces the reallocation of workers to more productive firms. ${ }^{9}$

Tilly and Niedermayer (2016) analyze employment and welfare effects of STC with a different focus, developing a dynamic model with heterogeneous workers that successfully captures several micro-level facts concerning STC take-up in Germany. This focus on dynamics and heterogeneity comes at the cost of assuming single-worker firms. Thus they do not consider a central feature in BW's and our analysis: multi-worker firms deciding how to spread reductions in total hours across layoffs and work sharing. A second key difference concerns private insurance. They argue that for Germany it is realistic to restrict employment contracts to an hourly wage, which furthermore cannot respond to temporary shocks in ongoing jobs. While employers are risk neutral, this limits their ability to insure workers. This inefficiency may explain why they find an optimal STC replacement rate close to $100 \%$ in their model.

\footnotetext{
${ }^{8}$ When firms lack access to perfect PI, an additional source of welfare gains from UI and potentially STC in our model is insurance provision against idiosyncratic profitability shocks. In contrast, in both BW and WH shocks are aggregate and thus undiversifiable, whether through public or private insurance.

${ }^{9}$ Cooper et al. (2016) find that short-time work (STW) reduces allocative efficiency, using a search model with heterogenous multi-worker firms and risk neutral workers. They model the policy instrument of STW as follows: in the absence of STW it is not permitted to reduce hours per worker below the normal level; STW eliminates this restriction. Thus STW is distinct from the policy instrument of STC we study in this paper. While they quantify the fiscal burden the government would face if it compensates workers for STW hours, they do not study the effects of STC on labor input decisions.
} 
Our contribution to the broader implicit contracts literature is the comparative statics property discussed above, which applies when firms lack access to PI and public UI is available: if profitability is sufficiently low for layoffs to be optimal, then a firm responds to a further reduction in profitability by reducing employment and increasing hours per worker. Rosen (1985) and FitzRoy and Hart (1985) study the corresponding comparative statics for the case of perfect PI, and show that hours are constant across profitability levels for which layoffs are optimal. The analysis closest to ours is Miyazaki and Neary (1985), who study the comparative statics of employment and hours for a firm without access to PI. They find that an increase in profitability can reduce both employment and hours per worker if firms have to cover fixed costs that are independent of employment, or if income effects are sufficiently strong. Our finding differs in that a change in profitability induces an opposite response of employment and hours, and that this pattern is induced by the presence of UI, which acts like a fixed cost per worker.

Blanchard and Tirole (2008) use a mechanism design approach to study optimal UI in a model with constant hours per worker. The key friction is that profitability is private information of the employer. They find that constrained efficiency generally requires that public insurance is exclusive, that is, supplementary UI provided by employers must be restricted. In contrast, we restrict the government to the instruments of UI and STC described above, and supplementary UI (and STC) by firms is unrestricted. It would be interesting to generalize Blanchard and Tirole's mechanism design approach to a setting with variable hours. Blanchard and Tirole also study optimal UI when firms' access to financial markets is limited, specifically by having shallow pockets. This resembles our case of imperfect PI, but the implications in their setting are quite different. ${ }^{10}$

The remainder of the paper is organized as follows. We introduce the model in Section 2. In Section 3 we characterize the allocation for a given system of UI and STC. Section 4 contains the computational experiments. Section 5 considers an alternative specification of technology, and Section 6 concludes.

\section{Model}

There is a continuum of firms, each with a mass $N$ of workers attached and jointly owned and operated by these workers. We normalize $N=1$. A fraction $v$ of the total population of workers is unattached.

Technology. Each firm has the production function $x f(n h)$ where $n$ denotes the mass of workers working strictly positive hours, $h$ denotes the number of hours worked by each of these workers, and

\footnotetext{
${ }^{10}$ Exclusivity together with the absence of worker moral hazard implies that workers are always fully insured in the constrained-efficient allocation, in the sense that the marginal utility of consumption is constant. In our setting, restricted policy instruments prevent the government from providing full insurance when firms lack access to perfect PI.
} 
$x$ parametrizes the profitability of the firm. The function $f:[0,+\infty) \rightarrow[0,+\infty)$ is twice continuously differentiable with $f^{\prime}>0$ and $f^{\prime \prime}<0$ on $(0,+\infty)$, and satisfies the Inada conditions $\lim _{l \rightarrow 0} f^{\prime}(l)=+\infty$ and $\lim _{l \rightarrow \infty} f^{\prime}(l)=0$. Profitability $x$ is subject to stochastic shocks that can be of technological or other origin, with density $p(x)$ and support $(0,+\infty)$.

Hours per worker and employment enter multiplicatively, thus hours of different workers are perfect substitutes. This specification is used by WH, and dubbed the standard case by BW. BW also study a specification with imperfect substitutability. We maintain the standard case for most of our analysis. In Section 5 we consider the case in which hours of different workers are perfect complements.

Preferences. The utility function of a worker is $\mathbb{E}[u(c, h)]$, where $c$ denotes consumption and $h$ denotes hours worked. The function $u$ takes the form proposed by King et al. (1988, KPR):

$$
u(c, h)=\frac{[c v(h)]^{1-\sigma}-1}{1-\sigma}
$$

with $\sigma>1$. The function $v:\left[0, h_{\max }\right) \rightarrow(0,1]$ satisfies $v(0)=1$. Here $h_{\max } \in(0,+\infty]$ is a physical upper limit on hours. The function $v$ incorporates a fixed utility loss from working strictly positive hours: $\lim _{h \rightarrow 0} v(h)=v_{0}$ with $v_{0} \in(0,1)$. The function $v$ is twice continuously differentiable and satisfies $v^{\prime}<0$ on $\left(0, h_{\max }\right)$. We assume that $-\frac{v^{\prime}}{v}$ is strictly increasing on $\left(0, h_{\max }\right)$ to ensure that consumption is a normal good. Let $V(h) \equiv-v(h)^{\frac{1-2 \sigma}{\sigma}} v^{\prime}(h)$. We assume $V^{\prime}(h)>0$ to ensure that $u(c, h)$ is strictly concave, and we impose the Inada condition $\lim _{h \rightarrow h_{\max }} V(h)=+\infty$.

Our specification is more general than BW in that we allow for a fixed utility loss from working strictly positive hours. It is less general than BW in that the KPR functional form restricts the relative strength of income and substitution effects. The KPR functional form is standard in macroeconomic models, since it is necessary for balanced growth. We see this paper as a step towards incorporating STC in a dynamic macroeconomic model, making this functional form a natural choice.

Private Insurance. We consider two polar cases, parametrized by $\chi \in\{0,1\}$. If $\chi=0$, firms have access to perfect PI. If $\chi=1$, firms have no access to PI.

Policy Instruments. UI takes the form of a payment $g_{U I}>0$ to workers with zero hours worked. STC takes the form of a payment $g_{S T C} \geq 0$ to employed workers for every hour that hours worked fall short of some normal level $\bar{h}$. We impose the restriction $\bar{h} g_{S T C} \leq g_{U I}$, so the maximal amount of STC, obtained by working marginally positive hours, cannot exceed the level of UI. The normal level $\bar{h}$ is taken as given by firms and equal to the average level of hours in equilibrium. Most countries with STC 
schemes require a minimum hours reduction (MHR). To capture this feature, firms are eligible for STC if hours are below $g_{M H R} \bar{h}$, where $g_{M H R} \leq 1$. The government balances the budget through a proportional $\operatorname{tax} \tau>0$ on total hours $n h$. Thus a firm with employment $n$ and hours $h$ receives the net subsidy

$$
(1-n) g_{U I}+n \mathcal{I}\left[h \leq g_{M H R} \bar{h}\right] \cdot(\bar{h}-h) \cdot g_{S T C}-\tau n h
$$

where $\mathcal{I}$ denotes the indicator function. Unattached workers receive the UI benefit $g_{U I}$. Notice that this system of UI and STC is uniform: it does not differentially treat workers based on the profitability of their firm, nor does it distinguish between attached and unattached workers. We do not model the reasons why the government does not use differential benefits.

This policy specification is based on $\mathrm{BW}$ and $\mathrm{WH}$, and generalizes theirs in three ways. First, they simplify the analysis by assuming that the normal level of hours $\bar{h}$ coincides with the physical upper limit $h_{\max }$. This implies that in their models firms always receive STC, allowing them to ignore the decision of whether to take up STC. We allow $\bar{h}$ and $h_{\max }$ to differ. To pin down $\bar{h}$ we require that in equilibrium it equals the average level of hours across states of the world. Second, BW restrict attention to two regimes: an American regime with $g_{S T C}=0$, and a European regime in which UI and STC are equally generous, that is, $\bar{h} g_{S T C}=g_{U I}$. We allow any value of $g_{S T C}$ between 0 and equal generosity. While many countries have equal replacement rates for UI and STC, in some countries STC is effectively less generous. For example, German firms pay social security contributions for hours not worked due to take-up of STC. In our computational experiments it turns out that equal generosity is not optimal. Third, in their specification firms receive STC whenever hours are below the normal level $\bar{h}$, which, as discussed above, coincides with the physical upper limit $h_{\max }$ in their model. We introduce the parameter $g_{M H R}$ to investigate whether a minimum hours reduction is a desirable feature of STC schemes.

BW assume that the government balances the budget through a lump sum tax. In their setup without a relevant eligibility threshold, this is isomorphic to our specification with a proportional tax on total hours. ${ }^{11}$ This is no longer true in our setup with an eligibility threshold. Given this, we prefer the proportional tax, since it mimics more closely the observed financing of UI through payroll taxes. ${ }^{12}$

Our specification does not include so-called experience rating, which requires that a firm reimburses the government for part of the UI and STC benefits received by its workers. Exactly as in the models

11 Without the eligibility threshold, net subsidy schedule $(2)$ reduces to $(1-n) g_{U I}+n(\bar{h}-h) \cdot g_{S T C}-\tau n h$. A system with UI benefit $\hat{g}_{U I}$ and STC benefit $\hat{g}_{S T C}$ financed through a lump sum tax $\hat{\tau}$ has the net subsidy schedule $(1-n) \hat{g}_{U I}+n(\bar{h}-h) \cdot \hat{g}_{S T C}-\hat{\tau}$. The isomorphism is defined by setting $\hat{g}_{U I}=g_{U I}+\bar{h} \tau, \hat{g}_{S T C}=g_{S T C}+\tau$, and $\hat{\tau}=\bar{h} \tau$.

${ }^{12}$ Of course a payroll tax would be based on wages. Thus it would not only depend on total hours, but also on profitability. We exclude policy instruments that condition on profitability. 
of $\mathrm{BW}$ and $\mathrm{WH}$, experience rating is redundant in our model: in Appendix $\mathrm{C}$ we show that a system with experience rating is equivalent to a system without experience rating and lower benefits. Thus we can omit experience rating without loss of generality. When mapping the model to the data, $g_{U I}$ and $g_{S T C}$ should be interpreted as subsidies net of any experience rating. Furthermore, the restriction that UI and STC are uniform should be understood as a restriction on net subsidies. ${ }^{13}$

Firm Optimization Problem. Let $T(x) \in\{0,1\}$ indicate the decision of the firm to take up STC in state $x$. Let $\iota(x)$ denote the net transfer received from PI in state $x$. The firm chooses $c_{w}(x), c_{b}(x)$, $n(x), h(x), \iota(x)$, and $T(x)$ for all $x \in(0,+\infty)$ to maximize

$$
\int_{0}^{\infty}\left\{n(x) u\left(c_{w}(x), h(x)\right)+(1-n(x)) u\left(c_{b}(x), 0\right)\right\} p(x) d x
$$

subject to

$$
\begin{aligned}
n(x) c_{w}(x)+(1-n(x)) c_{b}(x) & =x f(n(x) h(x))+\iota(x)-\tau n(x) h(x) \\
+(1-n(x)) g_{U I} & +n(x)(\bar{h}-h(x)) T(x) g_{S T C}, \\
n(x) & \leq 1, \\
T(x) \cdot\left(h(x)-g_{M H R} \bar{h}\right) & \leq 0, \\
\chi \iota(x) & =0
\end{aligned}
$$

for all $x \in(0,+\infty)$ and

$$
\int_{0}^{\infty} \iota(x) p(x) d x=0
$$

Constraint (8) requires that PI is actuarially fair. If $\chi=1$, then (7) enforces that the firm has no access to PI by requiring $\iota(x)=0$ in every state.

Government Optimization Problem. We restrict the government to choose the vector of policy instruments $g=\left\{g_{U I}, g_{S T C}, g_{M H R}, \bar{h}, \tau\right\}$ from a set $\mathcal{G}$. By varying $\mathcal{G}$, we can restrict the set of policy instruments available to the government. In our computational experiments, we consider a sequence of expanding sets $\mathcal{G}$, to examine the added value of introducing the policy instrument STC with and

\footnotetext{
${ }^{13}$ If only gross benefits are restricted to be uniform, and if experience rating is allowed to differentiate between workers based on profitability or attached status, then the restriction has no content, since any desired differentiation can be implemented through experience rating.
} 
without a minimum hours requirement. The objective function of the government is utilitarian welfare, giving weight $v$ to unattached workers. ${ }^{14}$ Let $U(g)$ denote the maximized value of the firm optimization problem as a function of the policy vector, and let $c_{w}(x, g), c_{b}(x, g), n(x, g), h(x, g), \iota(x, g)$, and $T(x, g)$ denote corresponding maximizers. Given these functions, the government chooses $g \in \mathcal{G}$ to maximize

$$
(1-v) U(g)+v u\left(g_{U I}, 0\right)
$$

subject to the government budget constraint

$$
\int_{0}^{\infty}\left\{(1-n(x, g)) g_{U I}+n(x, g)(\bar{h}-h(x, g)) T(x, g) g_{S T C}-\tau n(x, g) h(x, g)\right\} p(x) d x=0
$$

and the constraint that normal hours coincide with average hours per worker

$$
\bar{h}=\frac{\int_{0}^{\infty} n(x, g) h(x, g) p(x) d x}{\int_{0}^{\infty} n(x, g) p(x) d x} .
$$

First-Best Optimization Problem. A useful reference point for the allocations chosen by the government is the first-best allocation. It is obtained by choosing $c_{w}(x), c_{b}(x), n(x), h(x)$, and unattached workers' consumption $c_{\nu}$ to maximize utilitarian welfare

$$
(1-\nu) \int_{0}^{\infty}\left\{n(x) u\left(c_{w}(x), h(x)\right)+(1-n(x)) u\left(c_{b}(x), 0\right)\right\} p(x) d x+\nu u\left(c_{\nu}, 0\right)
$$

subject to constraint (5) and the resource constraint

$$
(1-\nu) \int_{0}^{\infty}\left\{n(x) c_{w}(x)+(1-n(x)) c_{b}(x)-x f(n(x) h(x))\right\} p(x) d x+\nu c_{\nu}=0 .
$$

If firms have access to perfect PI, the only reason why the government cannot achieve the first best is that attached workers on layoff are not excluded from UI. If firms lack access to PI, then a second reason is that its policy instruments do not permit conditioning transfers directly on profitability $x$.

\section{Optimal Firm Behavior}

In this section we analyze the firm optimization problem, proceeding in three steps. In Section 3.1 we derive first-order conditions and obtain comparative statics properties of optimal hours. In Section 3.2

\footnotetext{
${ }^{14}$ One can also interpret attachment as an initial uninsurable shock. In this interpretation all workers are ex ante identical, and the government simply maximizes expected utility.
} 
we analyze how optimal labor inputs vary with profitability conditional on the decision to take up STC. That is, we fix the take-up decision, and study optimal labor input profiles separately for the cases of take-up and no take-up of STC. In Section 3.3 we combine these results to discuss the take-up decision.

\subsection{First-Order Conditions}

Let $\lambda(x) p(x), \nu(x) p(x), \zeta(x) p(x), \rho(x) p(x)$, and $\mu$ denote the multipliers associated with constraints (4), (5), (6), (7), and (8). The first-order conditions for $c_{w}(x), c_{b}(x), n(x), h(x)$, and $\iota(x)$ are

$$
\begin{aligned}
& u_{c}\left(c_{w}(x), h(x)\right)=\lambda(x), \\
& u_{c}\left(c_{b}(x), 0\right)=\lambda(x), \\
& u\left(c_{b}(x), 0\right)-u\left(c_{w}(x), h(x)\right)=\lambda(x)\left[x f^{\prime}(n(x) h(x)) h(x)-c_{w}(x)+c_{b}(x)\right.\left.-g_{U I}+(\bar{h}-h(x)) T(x) g_{S T C}-\tau h(x)\right]-\nu(x), \\
&-n(x) u_{h}\left(c_{w}(x), h(x)\right)=\lambda(x)\left[x f^{\prime}(n(x) h(x)) n(x)-n(x) T(x) g_{S T C}-\tau n(x)\right]-T(x) \zeta(x), \\
& \lambda(x)=\mu+\rho(x) \chi .
\end{aligned}
$$

Conditions (12)-(13) imply that consumption levels of employed and unemployed workers are $c_{w}(x)=$ $c_{w}^{*}(\lambda(x), h(x))$ and $c_{b}(x)=c_{b}^{*}(\lambda(x))$, respectively, with $c_{w}^{*}(\lambda, h) \equiv \lambda^{-1 / \sigma} v(h)^{(1-\sigma) / \sigma}$ and $c_{b}^{*}(\lambda) \equiv \lambda^{-1 / \sigma}$.

Next, we analyze the first-order conditions that determine the optimal level of hours per worker. We first consider the case in which the employment constraint (5) is slack, and then turn to the case in which it binds. In both cases we focus on the case in which constraint (6) is slack, since its impact on optimal hours is straightforward. If the constraints (5) and (6) are slack, that is, if $\nu(x)=0$ and $\zeta(x)=0$, then combining first-order conditions (14) and (15) yields

$$
u\left(c_{b}(x), 0\right)-u\left(c_{w}(x), h(x)\right)+u_{h}\left(c_{w}(x), h(x)\right) h(x)=\lambda(x)\left[c_{b}(x)-c_{w}(x)-g_{U I}+\bar{h} \cdot T(x) g_{S T C}\right] .
$$

This is the first-order condition for a variation that reduces employment while increasing hours per worker $h$ to keep total hours $n h$ constant. The left-hand side gives the utility gain from this variation. Each worker now has a larger chance of being on layoff, which yields the utility gain $u\left(c_{b}(x), 0\right)-u\left(c_{w}(x), h(x)\right)$. To keep total hours constant, the additional layoff must be compensated by redistributing $h(x)$ hours across the remaining workers, which yields a utility loss of $-u_{h}\left(c_{w}(x), h(x)\right) h(x)$. The right-hand side gives the impact of this variation on the budget constraint. The additional worker on layoff is switched from consumption $c_{w}(x)$ to consumption $c_{b}(x)$ and collects the UI benefit $g_{U I}$. The firm loses $(\bar{h}-h(x))$. 
$T(x) g_{S T C}$ in STC for the worker on layoff, and an additional $h(x) \cdot T(x) g_{S T C}$ due to higher hours for remaining workers, for a total of $\bar{h} \cdot T(x) g_{S T C}$.

Substituting the functions $c_{w}^{*}$ and $c_{b}^{*}$, we obtain a condition linking hours and the multiplier $\lambda$ which does not directly involve profitability $x$ :

$$
u\left(c_{b}^{*}(\lambda), 0\right)-u\left(c_{w}^{*}(\lambda, h), h\right)+u_{h}\left(c_{w}^{*}(\lambda, h), h\right) h+\lambda\left[c_{w}^{*}(\lambda, h)-c_{b}^{*}(\lambda)+g_{U I}-\bar{h} T \cdot g_{S T C}\right]=0 .
$$

The following proposition establishes that this equation has a unique solution for hours, and characterizes the comparative statics of hours with respect to $\lambda$ and $T$. All proofs are collected in Appendix A.

Proposition 1 Equation (18) has a unique solution for $h$ given any $\lambda>0$ and $T \in\{0,1\}$. If $g_{S T C}>0$, then this solution is strictly decreasing in T. If $g_{U I}-\bar{h} T \cdot g_{S T C}>0$, then it is strictly increasing in $\lambda$. If $g_{U I}-\bar{h} T \cdot g_{S T C}=0$, then it is independent of $\lambda$.

Hours are decreasing in $T$ if $g_{S T C}>0$, since $g_{S T C}$ subsidizes low hours. The relationship between the multiplier $\lambda$ and hours is less obvious. In the absence of a net payment from the government $\left(g_{U I}-\bar{h} T \cdot g_{S T C}=0\right)$, hours are determined by the trade-off between the fixed disutility of working positive hours and the increasing marginal disutility of working long hours. Higher fixed costs favor longer hours, while convex disutility favors spreading hours across many workers. With KPR utility, the optimal level of hours determined by this trade-off is not affected by the multiplier $\lambda$. UI benefits introduce an additional fixed cost of working positive hours, incurred in terms of the consumption good. A higher multiplier $\lambda$ indicates that consumption is more valuable. This shifts the trade-off in favor of higher hours. Thus UI distorts the composition of labor inputs in the direction of higher hours and lower employment. If taken up, STC counteracts this distortion and eliminates it entirely if UI and STC are equally generous, that is, if $\bar{h} g_{S T C}=g_{U I}$. The property that hours are strictly increasing in $\lambda$ if $g_{U I}-\bar{h} T \cdot g_{S T C}>0$ and independent of $\lambda$ if $g_{U I}-\bar{h} T \cdot g_{S T C}=0$ also holds for other common specifications of utility. In particular, it also holds when utility is additively separable in consumption and hours. With GHH preferences, hours are independent of $\lambda$ even if $g_{U I}-\bar{h} T \cdot g_{S T C}>0 .{ }^{15}$

The key implication of equation (18) is that hours are affected by profitability $x$ only through the multiplier $\lambda(x)$, which is the marginal utility of consumption. With perfect PI, $\lambda(x)$ does not vary with profitability, hence hours are constant. As we discuss below, without PI $\lambda(x)$ is decreasing in $x$, hence hours are declining in $x$. Thus firms experiencing an uninsured decline in profitability and engaging in layoffs have relatively high hours for those workers that remain at work.

\footnotetext{
${ }^{15}$ These claims are established at the end of the proof of Proposition 1.
} 
Next, consider the case in which the employment constraint is binding. Substituting $n(x)=1$ along with the function $c_{w}^{*}$ into first-order condition (15) yields

$$
-u_{h}\left(c_{w}^{*}(\lambda, h), h\right)=\lambda\left[x f^{\prime}(h)-\tau-T \cdot g_{S T C}\right]
$$

Substituting the functional forms of $u_{h}$ and $c_{w}^{*}$ yields

$$
V(h)=\lambda^{\frac{1}{\sigma}}\left[x f^{\prime}(h)-\tau-T \cdot g_{S T C}\right]
$$

The following proposition establishes that this equation has a unique solution for hours, and characterizes the comparative statics of hours with respect to $x, \lambda$, and $T$.

Proposition 2 Equation (19) has a unique solution for $h$ given any $x>0$ and $T \in\{0,1\}$. This solution is strictly increasing in $x$ and $\lambda$, and converges to $h_{\max }$ as $x$ converges to infinity. If $g_{S T C}>0$, then it is strictly decreasing in $T$.

UI does not directly affect the choice of hours when the firm does not engage in layoffs.

\subsection{Labor Input Profiles Conditional on STC Take-Up}

In this section we analyze how optimal labor inputs vary with profitability conditional on STC take-up, separately for the cases of perfect PI and no PI. Let $h^{0}(x)$ and $n^{0}(x)$ denote the levels of hours and employment that would be optimal if STC is not taken up. Here the superscript indicates that $T=0$. Analogously, let $h^{1}(x)$ and $n^{1}(x)$ denote the corresponding levels if STC is taken up, that is, if $T=1$.

\subsubsection{Perfect Private Insurance}

Proposition 3 If $\chi=0$, then the functions $h^{0}(x), n^{0}(x), h^{1}(x)$, and $n^{1}(x)$ are continuous and have the following properties.

1. There exists a threshold $x_{N}^{0} \in(0,+\infty)$ such that $h^{0}(x)$ is constant on $\left(0, x_{N}^{0}\right)$ and strictly increasing on $\left(x_{N}^{0},+\infty\right)$, while $n^{0}(x)$ is strictly increasing on $\left(0, x_{N}^{0}\right)$ and equal to one on $\left(x_{N}^{0},+\infty\right)$.

2. There exist thresholds $x_{N}^{1} \in(0,+\infty)$ and $x_{M H R}^{1} \in\left[x_{N}^{1},+\infty\right]$ such that $h^{1}(x)$ is constant on $\left(0, x_{N}^{1}\right)$, strictly increasing on $\left(x_{N}^{1}, x_{M H R}^{1}\right)$, and constant at $g_{M H R} \bar{h}$ on $\left(x_{M H R}^{1},+\infty\right)$, while $n^{1}(x)$ is strictly increasing on $\left(0, x_{N}^{1}\right)$ and equal to one on $\left(x_{N}^{1},+\infty\right)$.

3. If $g_{S T C}>0$, then $h^{1}(x)<h^{0}(x)$ for all $x \in(0,+\infty)$. 
Figure 1: Labor Input Profiles and STC Take-Up with Perfect PI

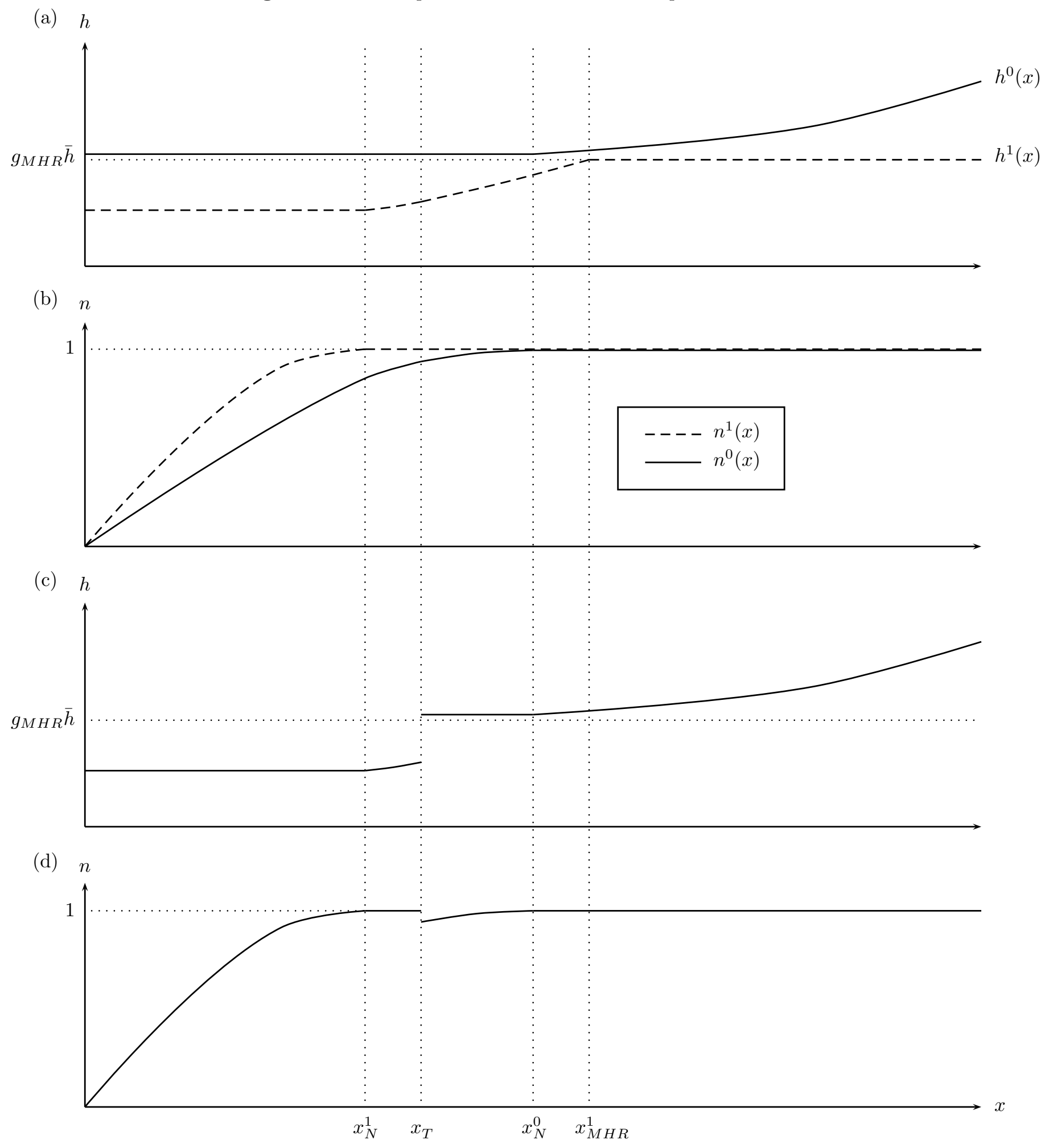


This proposition is illustrated in Panels (a) and (b) of Figure 1. Part 1 characterizes $h^{0}(x)$ and $n^{0}(x)$. There are two profitability regions across which the qualitative behavior of labor inputs differs, divided by a threshold $x_{N}^{0}$ at which the employment constraint becomes binding. Below this threshold the firm engages in layoffs, and hours per workers are constant. The latter follows directly from Proposition 1 , which states that hours do not vary with profitability $x$ conditional on the multiplier $\lambda(x)$. Perfect PI implies that $\lambda(x)$ is independent of $x$, hence hours are constant. Employment is strictly increasing over this region. Above $x_{N}^{0}$ the behavior of hours is governed by Proposition 2. Hours are now strictly increasing in profitability as it is no longer possible to take advantage of higher profitability by raising employment. This characterization of labor input profiles in the case of perfect PI is well-known, and can be found in Rosen (1985), FitzRoy and Hart (1985), and Burdett and Wright (1989), among others.

Part 2 of the proposition describes $h^{1}(x)$ and $n^{1}(x)$. Again there is a threshold $x_{N}^{1}$ at which the employment constraint becomes binding, and the qualitative behavior of labor inputs above and below this threshold is very similar to the case of no take-up. The only difference stems from the MHR constraint. Above $x_{N}^{1}$, hours are strictly increasing in profitability until the MHR constraint is binding. It is also possible that the MHR constraint is already binding below $x_{N}^{1}$, in which case hours do not vary with profitability over the entire profitability range $(0,+\infty)$.

Part 3 shows that hours under take-up are always below hours under no take-up. In essence, this follows directly from the comparative statics for hours with respect to take-up established in Propositions 1 and $2 .{ }^{16}$ Part 3 is silent on the relative position of the employment schedules $n^{0}(x)$ and $n^{1}(x)$. Firstorder condition (14) shows that take-up provides an employment subsidy of $(\bar{h}-h) g_{S T C}$ per worker, which by itself increases employment. The effect of take-up on employment is ambiguous, however, as the reduction in hours induced by take-up reduces the marginal product from employing an additional worker. ${ }^{17}$ In particular, it is ambiguous whether $n^{0}(x)$ or $n^{1}(x)$ attains one first, that is, the relative position of the thresholds $x_{N}^{0}$ and $x_{N}^{1}$ is also ambiguous. In our computational experiments the case $n^{1}(x)>n^{0}(x)$ always prevails, which implies $x_{N}^{1}<x_{N}^{0}$. This case is illustrated in Panel (b) of Figure 1.

\subsubsection{No Private Insurance}

Proposition 4 If $\chi=1$, then the functions $h^{0}(x), n^{0}(x), h^{1}(x)$, and $n^{1}(x)$ are continuous and have the following properties.

1. There exists $x_{N}^{0} \in[0,+\infty]$ such that $h^{0}(x)$ is strictly decreasing on $\left(0, x_{N}^{0}\right)$ and strictly increasing

\footnotetext{
16 Proposition 1 implies this result for profitability below $\min \left[x_{N}^{0}, x_{N}^{1}\right]$, and Proposition 2 does so for the region above $\max \left[x_{N}^{0}, x_{N}^{1}\right]$. The only extra work in the proof of Part 3 of Proposition 3 is to establish this result between $x_{N}^{0}$ and $x_{N}^{1}$.

${ }^{17}$ Van Audenrode (1994, p. 84) notes this ambiguity in a similar model.
} 
Figure 2: Labor Input Profiles and STC Take-Up without PI

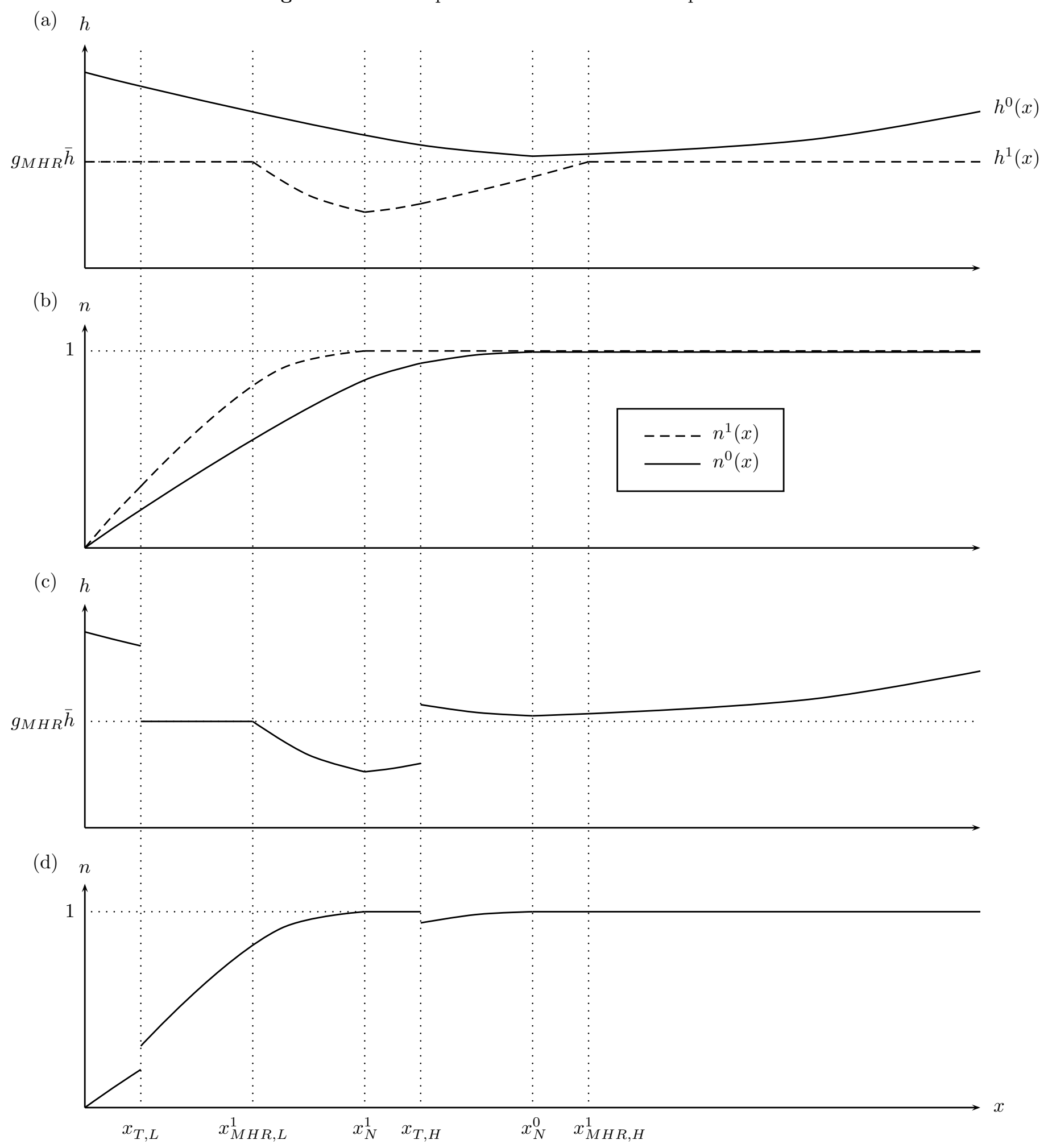


on $\left(x_{N}^{0},+\infty\right)$, while $n^{0}(x)$ is strictly increasing on $\left(0, x_{N}^{0}\right)$ and equal to one on $\left(x_{N}^{0},+\infty\right)$.

2. There exist $x_{N}^{1} \in[0,+\infty], x_{M H R, L}^{1} \in\left[0, x_{N}^{1}\right]$, and $x_{M H R, H}^{1} \in\left[x_{N}^{1},+\infty\right]$ such that $h^{1}(x)$ is constant at $g_{M H R} \bar{h}$ on $\left(0, x_{M H R, L}^{1}\right)$, weakly decreasing on $\left(x_{M H R, L}^{1}, x_{N}^{1}\right)$, strictly increasing on $\left(x_{N}^{1}, x_{M H R, H}^{1}\right)$, and constant at $g_{M H R} \bar{h}$ on $\left(x_{M H R, H}^{1},+\infty\right)$. It is strictly decreasing on $\left(x_{M H R, L}^{1}\right.$, $\left.x_{N}^{1}\right)$ if $g_{U I}-\bar{h} g_{S T C}>0 . n^{1}(x)$ is strictly increasing on $\left(0, x_{N}^{1}\right)$ and equals one on $\left(x_{N}^{1},+\infty\right)$.

3. If $g_{S T C}>0$, then $h^{1}(x)<h^{0}(x)$ for all $x \in(0,+\infty)$.

This proposition is illustrated in Panels (a) and (b) of Figure 2. Employment schedules behave qualitatively as in the case of perfect PI. In contrast, the behavior of hours is different. Consider first the case of no take-up. The profile $h^{0}(x)$ is strictly decreasing below the threshold $x_{N}^{0}$. This is explained by Proposition 1, according to which hours are strictly increasing in the multiplier $\lambda$ if $g_{U I}-\bar{h} T \cdot g_{S T C}>0$, which holds if STC is not taken up. In the absence of PI the multiplier $\lambda$, which coincides with marginal utility of consumption, is strictly decreasing in $x{ }^{18}$ This carries over to hours. As explained in the discussion of Proposition 1, $\lambda$ affects optimal hours through its interaction with $g_{U I}$, which acts like a fixed cost of employment in terms of the consumption good. Consumption is scarce after an uninsured decline in profitability. The optimal response of the firm is to send workers to collect UI benefits, which is one way of obtaining consumption, and to implement longer hours for workers that remain on the job. ${ }^{19}$ This comparative statics result is new to the implicit contracts literature. ${ }^{20}$ In Section 4 we show that it has important implications for the welfare effects of STC.

Hours are strictly increasing in $x$ above the full-employment threshold $x_{N}^{0}$. Qualitatively, this is as in the case of perfect PI, but the economic forces are somewhat different. With perfect PI, the increase in hours is purely driven by a substitution effect, thus our assumption of KPR preferences is not important for this result. In contrast, here the marginal utility of consumption is decreasing in profitability, hence the response of hours depends on the relative strength of income and substitution effects. With KPR preferences these effects would cancel exactly in the absence of policy, that is, if $\tau=0$. A positive tax $\tau$ makes the income effect relatively weaker, hence the substitution effect dominates.

The hours profile conditional on take-up of STC $h^{1}(x)$ is qualitatively similar to $h^{0}(x)$. As in the case of perfect PI, its shape only differs due to the MHR constraint. However, the hours profile would be $V$-shaped in the absence of the MHR constraint. This implies that in general there are two profitability

\footnotetext{
${ }^{18}$ This is established in the course of the proof of Proposition 4.

${ }^{19}$ Notice that we have assumed that all fixed costs of employment accrue in terms of utility, so that UI is the only fixed cost in terms of consumption. If other fixed costs also accrue in terms of consumption, then this strengthens the result.

${ }^{20}$ As discussed in Footnote 6, this result continues to apply if the roles of workers and employers are separated.
} 
intervals over which the MHR constraint binds. First, below a threshold $x_{M H R, L}^{1}$, which lies in the profitability range with a slack employment constraint. Second, above a threshold $x_{M H R, H}^{1}$, which lies in the profitability range over which the employment constraint binds.

Part 3 establishes that, as in the case of perfect PI, take-up reduces hours.

\subsection{STC Take-Up}

Having analyzed labor input profiles conditional on take-up, we now discuss optimal take-up. Consider first the case of perfect PI. The next proposition gives sufficient conditions such that take-up is monotone in profitability, occurring at low levels of profitability.

Proposition 5 Suppose that $\chi=0$ and $g_{S T C}>0$. If $f(n h)=(n h)^{\alpha}$ with $\alpha \in(0,1)$ and $x_{N}^{1}<x_{N}^{0}$, then there exists $x_{T} \in[0,+\infty]$ such that optimal take-up is $T^{*}(x)=1$ on $\left(0, x_{T}\right]$ and $T^{*}(x)=0$ on $\left(x_{T}, \infty\right)$.

The first condition is that the technology is Cobb-Douglas, which we employ in our computational experiments. The second condition is that the employment constraint starts to bind at a lower level of profitability in the case of take-up, that is, $x_{N}^{1}<x_{N}^{0}$. As discussed in the context of Proposition 3, $x_{N}^{1}<x_{N}^{0}$ prevails in all our computational experiments, although the reverse is a theoretical possibility.

The monotonicity of optimal take-up in Proposition 5 is driven by the complementarity between total hours $n^{T}(x) h^{T}(x)$ and profitability. Take-up is associated with a reduction in hours. Everything else equal, this leads to lower total hours. This can be countered by an increase in employment, but only if the employment constraint is slack. Once profitability is sufficiently high, firms taking up STC run into the employment constraint. This makes take-up more costly, the more so the higher is profitability.

The take-up threshold $x_{T}$ can lie anywhere in $[0,+\infty]$. Panels $(\mathrm{c})$ and $(\mathrm{d})$ of Figure 1 illustrate the optimal labor input profiles for the case in which $x_{T}$ lies between the two employment thresholds $x_{N}^{1}$ and $x_{N}^{0}$. They are generated from Panels (a) and (b) by selecting the take-up schedules $h^{1}(x)$ and $n^{1}(x)$ to the left of $x_{T}$, and the no take-up schedules $h^{0}(x)$ and $n^{0}(x)$ to the right of $x_{T}$. As $x$ increases, hours are first flat while employment increases. Hours start to increase as the employment constraint becomes binding under take-up at $x_{N}^{1}$. Next, hours jump up and employment jumps down at the take-up threshold $x_{T}$. After that, hours are once again flat while employment increases until the employment constraint becomes binding under no take-up at $x_{N}^{0}$. Beyond this point, hours are once again increasing.

Next, consider the case of no PI. Here we have no theoretical results for take-up, as the analysis is substantially complicated by income effects. As with perfect PI, one force is that take-up is more costly if higher hours would be optimal conditional on no take-up. With perfect PI, this gives rise to the following property: take-up is monotone in the hours that the firm would choose conditional on no-take 
up. Since the latter are monotone in $x$, so is take-up. For illustration, suppose that this force remains dominant in shaping take-up. The key difference to perfect PI is that hours are not monotone in $x$, but $V$-shaped. Thus one would expect no take-up to occur in two separate regions of profitability, both at very low and very high levels of $x$. Panels (c) and (d) of Figure 2 illustrate such a case with two take-up thresholds, denoted $x_{T, L}$ and $x_{T, H}$. The lower take-up threshold $x_{T, L}$ is located in the profitability region over which hours conditional on take-up are strictly declining in $x$, both for $T=1$ and $T=0$. In the case illustrated here, the second take-up threshold is located between $x_{N}^{1}$ and $x_{N}^{0}$, when hours are still strictly decreasing in $x$ conditional on no take-up, but are already strictly increasing in profitability conditional on take-up due to a binding employment constraint. The labor input schedules in Panels (c) and (d) are generated from Panels (a) and (b) by selecting the no-take schedules $h^{0}(x)$ and $n^{0}(x)$ to the left and to the right of $x_{T, L}$ and $x_{T, H}$, respectively, and the take-up schedules $h^{1}(x)$ and $n^{1}(x)$ in between. Hours jump down and employment jumps up at $x_{T, L}$, the reverse happens at $x_{T, H}$.

\section{Computational Experiments}

In this section we carry out computational experiments to examine whether introducing STC can improve on a system restricted to UI in our model. We obtain two main results. First, whether STC can improve on UI depends critically on firms' access to PI. STC substantially improves welfare if firms have access to perfect PI, but yields only a negligible improvement when firms lack access to PI. Under perfect PI, STC improves welfare by reducing excessive layoffs induced by UI. This mechanism is greatly diminished if firms lack access to PI, because the most distressed firms prefer long hours over taking up STC. Second, the optimal generosity of STC is substantially below that of UI even with perfect PI, and introducing STC with equal generosity results in a large welfare loss in comparison to having no STC at all.

\subsection{Calibration}

We calibrate the model to match features of the US labor market. The functional form of $f$ is

$$
f(n h)=(n h)^{\alpha} .
$$

We set $\alpha=\frac{2}{3}$, implicitly assuming that capital cannot be adjusted in response to profitability shocks. For the utility function given in equation (1) above, we specify

$$
v(h)=\exp \left(-\eta \frac{h^{1+\psi}}{1+\psi}+\log \left(v_{0}\right) \mathcal{I}[h>0]\right)
$$


where $\eta$ and $\psi$ are strictly positive. The parameter $\eta$ only affects the level of hours, so we can use it to normalize employment-weighted average hours to one. We set the coefficient of relative risk aversion to $\sigma=2$, within the "plausible" range 1-5 indicated by micro estimates, see Heathcote et al. (2009). The parameter $\psi$ governs the Frisch elasticity of labor supply. Based on the recent survey of the microeconomic evidence in Hall (2009), we target a Frisch elasticity of $0.7 .^{21}$ We set $v=0.045$, so that $4.5 \%$ of workers are not attached to a firm. Together with the level of temporary layoffs targeted below, this matches the average unemployment rate in the US of about $6 \%$.

The density $p(x)$ is $\log$-normal. We normalize the mean of $\log (x)$ to zero and set its standard deviation to $\sigma_{x}=0.1$, a reasonable order of magnitude for firm-specific shocks for a time horizon between six months and one year, see for example Comin and Philippon (2006) and Davis et al. (2007).

We calibrate an economy that has UI but no STC. Thus two parameters remain to be calibrated: $v_{0}$, which governs the fixed utility loss from working strictly positive hours, and the UI benefit $g_{U I}$. We jointly calibrated them to match two targets. First, we target that $1.5 \%$ of all workers experience a temporary layoff. Thus $25 \%$ of all the unemployed are attached. We base this target on the empirical prevalence of temporary layoffs. In the US Current Population Survey, on average 14\% of the stock of unemployed workers is classified as on temporary layoff. ${ }^{22,23}$ Fujita and Moscarini (2016) find that temporary layoffs are relatively more important for flows, accounting for one third of the flow from employment to unemployment. Our target strikes a balance between the importance of temporary layoffs for flows and stocks, as our static model cannot match them separately. Second, we target a UI replacement rate of $25 \%$, where we define the replacement rate in the model as $g_{U I}$ divided by the average consumption of workers. Recall that experience rating is neutral in our model and $g_{U I}$ corresponds to the UI subsidy net of experience rating. Topel (1983) reports that on average the net subsidy is $31 \%$ of earnings. In our model workers jointly own and operate firms, hence implicitly their average consumption reflects income from both wages and profits. This leads us to adopt the somewhat lower target of $25 \%$. These targets pin down $g_{U I}$ and $v_{0}$ as follows. Both $g_{U I}$ and $v_{0}$ act as a fixed cost of working positive hours. The fraction of workers on temporary layoff is increasing in fixed costs, so the corresponding target pins down $v_{0}$ for given $g_{U I}$. We then vary $g_{U I}$ to match the targeted replacement rate.

\footnotetext{
${ }^{21}$ The Frisch elasticity is $\left(\psi+\frac{\sigma-1}{\sigma}\left(\eta h^{1+\psi}\right)\right)^{-1}$. At average hours, this reduces to $\left(\psi+\frac{\sigma-1}{\sigma} \eta\right)^{-1}$.

${ }^{22}$ The average is taken over the years 1967-2012.

${ }^{23}$ Compared with other countries for which evidence is available, the incidence of temporary layoffs in the US is about average. In a survey of the available evidence, OECD (2002) reports that temporary layoffs account for almost $40 \%$ of unemployment in Canada, 20\% of unemployment in Austria and Denmark, and fractions closer to 10\% in other European countries such as Germany, Norway and Sweden.
} 
Table 1: Calibration: Perfect PI and No PI

\begin{tabular}{lccl}
\hline \hline Value & Perfect PI & No PI & Target \\
\hline$\sigma$ & 2 & 2 & \\
$\alpha$ & 0.67 & 0.67 & \\
$\sigma_{x}$ & 0.1 & 0.1 & \\
$\psi$ & 1.1 & 1.1 & Frisch elasticity 0.7 \\
$\eta$ & 0.664 & 0.667 & $\bar{h}=1$ (Normalization) \\
$v$ & 0.045 & 0.045 & Unattached workers 0.045 \\
$v_{0}, \% c, \% h$ & $0.934,6.6,9.8$ & $0.89,11,16$ & Temporary layoffs 0.015 \\
$g_{U I}$ & 0.247 & 0.247 & Replacement rate $25 \%$ \\
\hline \hline
\end{tabular}

The calibration for both cases, perfect PI and no PI, is summarized in Table 1. The policy parameter $g_{U I}$ is pinned down quite directly by the replacement rate target. Only the utility fixed cost $v_{0}$ differs substantially between the two calibrations. With perfect PI it equals 0.934 , which corresponds to $6.63 \%$ in terms of consumption and $9.76 \%$ in terms of hours. ${ }^{24}$ Its value is higher in the case of no PI, corresponding to $11 \%$ in terms of consumption. Lack of insurance makes firms more reluctant to carry out layoffs, thus the fixed cost must be higher to match the targeted level of temporary layoffs. In Appendix B we show that the main results obtained in the remainder of this section are insensitive to changes in parameters and targets over a wide range of values.

We carry out the following sequence of policy experiments, summarized in Table 2. Each experiment is defined by restrictions on the set of policy instruments $\mathcal{G}$ in the government optimization problem of Section 2. First, we restrict this set to UI and determine the welfare-maximizing level of $g_{U I}$. We denote this as $g_{U I}^{*}$, and also use $g_{U I}^{*}$ to label this experiment. We use $g_{U I}^{*}$ rather than the calibrated level of $g_{U I}$ as the starting point for experiments that introduce STC. Otherwise welfare gains from STC could merely reflect a suboptimal level of $g_{U I}$, rather than a genuine added value of $g_{S T C}$ as a policy instrument. The next three experiments introduce STC without a minimum hours requirement, hence $g_{M H R}=1$. In the first, we determine the optimal level of $g_{S T C}$ holding constant $g_{U I}$ at $g_{U I}^{*}$. By construction, introducing $g_{S T C}$ in this way does not affect the level of consumption of unattached workers. Therefore, to the extent that STC does improve the allocation, it can only do so by mitigating the distortion of labor inputs induced by $U I$. We refer to the corresponding level of STC and also the entire experiment as $g_{S T C}^{*} \mid g_{U I}^{*}$ to indicate that $g_{S T C}^{*}$ is optimal conditional on fixing the level of UI at $g_{U I}^{*}$. In the second experiment, we introduce a level of $g_{S T C}$ that is as generous as $g_{U I}^{*}$. This level satisfies $g_{S T C} \bar{h}=g_{U I}^{*}$, and the corresponding experiment is labeled $g_{S T C}^{\max } \mid g_{U I}^{*}$. In the next step, we determine the welfare-maximizing

\footnotetext{
${ }^{24}$ The cost associated with $v_{0}$ is expressed in terms of consumption (hours) by considering a compensating proportional decrease in consumption (increase in hours) that leaves workers with $v_{0}=0$ as well off as under the calibrated value.
} 
Table 2: Policy Experiments

\begin{tabular}{lll}
\hline \hline Policy Experiment & Restrictions on the Set of Policy Instruments $\mathcal{G}$ & Remarks \\
\hline$g_{U I}^{*}$ & $g_{S T C}=0, g_{M H R}=1$ & \\
$g_{S T C}^{*} \mid g_{U I}^{*}$ & $g_{U I}=g_{U I}^{*}, g_{M H R}=1$ & \\
$g_{S T C}^{m a x} \mid g_{U I}^{*}$ & $g_{U I}=g_{U I}^{*}, g_{S T C} \bar{h}=g_{U I}^{*}, g_{M H R}=1$ & Perfect PI only \\
$\left(g_{U I}, g_{S T C}\right)^{*}$ & $g_{M H R}=1$ & \\
$\left(g_{S T C}, g_{M H R}\right)^{*} \mid g_{U I}^{*}$ & $g_{U I}=g_{U I}^{*}$ & \\
$\left(g_{U I}, g_{S T C}, g_{M H R}\right)^{*}$ & None & \\
$\bar{g}_{S T C} \mid g_{U I}^{*}$ & $g_{U I}^{*}=g_{U I}^{*}, g_{S T C} \bar{h} / g_{U I}$ takes same value as in & No PI only \\
\hline \hline
\end{tabular}

Table 3: Policy Experiments: Perfect PI

\begin{tabular}{|c|c|c|c|c|c|c|c|c|}
\hline & Calibr. & 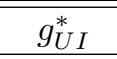 & $g_{S T C}^{*} \mid g_{U I}^{*}$ & $g_{S T C}^{\max } \mid g_{U I}^{*}$ & $\left(g_{U I}, g_{S T C}\right)^{*}$ & $\left(\bar{c}_{S T C}, g_{M H R}\right)^{*} \mid g_{U I}^{*}$ & $\left(\bar{c}_{U I}, g_{S T C}, g_{M H R}\right)^{*}$ & FB \\
\hline$g_{U I}$ & 0.247 & 0.262 & 0.262 & 0.262 & 0.284 & 0.262 & 0.28 & \\
\hline$g_{S T C}$ & & & 0.08 & 0.308 & 0.13 & 0.0643 & 0.0933 & \\
\hline$g_{M H R}$ & & & 1 & 1 & 1 & 0.81 & 0.82 & \\
\hline$\tau$ & 0.0158 & 0.0214 & 0.0196 & 0.0501 & 0.0278 & 0.017 & 0.0237 & \\
\hline$R E P R_{U I}(\%)$ & 25 & 26.7 & 27.1 & 29.2 & 29.9 & 26.8 & 29.1 & \\
\hline$R E P R_{S T C}(\%)$ & & & 7.97 & 29.2 & 13 & 6.43 & 9.32 & \\
\hline STC Take-Up (\%) & & & 51.6 & 49 & 52.1 & 16.2 & 28.1 & \\
\hline $\bar{n}$ & 0.984 & 0.968 & 0.988 & 1 & 0.98 & 0.991 & 0.982 & 1 \\
\hline $\bar{h}$ & 1 & 1 & 0.965 & 0.853 & 0.943 & 0.98 & 0.963 & 1.02 \\
\hline $\bar{y}$ & 0.999 & 0.991 & 0.979 & 0.911 & 0.96 & 0.991 & 0.975 & 1.02 \\
\hline $\bar{c}$ & 0.987 & 0.978 & 0.967 & 0.898 & 0.947 & 0.978 & 0.961 & 0.982 \\
\hline Welf. Rel. to $g_{U I}^{*}(\% c)$ & -0.1886 & & 0.30107 & -1.8582 & 0.52629 & 0.4027 & 0.60792 & 6.0165 \\
\hline
\end{tabular}


combination of $g_{S T C}$ and $g_{U I}$ denoting this experiment as $\left(g_{U I}, g_{S T C}\right)^{*}$. The next two experiments introduce an MHR by allowing $g_{M H R}$ do differ from one. First, in the experiment $\left(g_{S T C}, g_{M H R}\right)^{*} \mid g_{U I}^{*}$ we once again fix the level of $U I$ at $g_{U I}^{*}$ while jointly choosing $g_{S T C}$ and $g_{M H R}$ optimally. Finally, in the experiment $\left(g_{U I}, g_{S T C}, g_{M H R}\right)^{*}$ we choose all three policy instruments optimally.

\subsection{Perfect Private Insurance}

Results for the case of perfect PI are in Table 3. The calibration and the first best (FB) are shown as points of reference. For each experiment, the first six rows show the values of the policy instruments $g_{U I}, g_{S T C}$ and $g_{M H R}$, and the tax $\tau$, along with the replacement rates implied by $g_{U I}$ and $g_{S T C}$, labeled $R E P R_{U I}$ and $R E P R_{S T C}$, respectively. ${ }^{25}$ The next row reports the STC take-up rate, that is, the average fraction of attached workers receiving $S T C$ in percent. The next four rows show the average of employment and average (employment weighted) hours for attached workers, denoted $\bar{n}$ and $\bar{h}$, respectively, along with average output $\bar{y}$ and consumption $\bar{c}$ across attached workers. The final row shows, for each allocation, the welfare gain vis-à-vis the experiment $g_{U I}^{*}$. Here and in the remainder of the paper, welfare gains are expressed in percentage consumption-equivalent terms. Figure 3 compares labor input profiles for the three experiments $g_{U I}^{*}, g_{S T C}^{*} \mid g_{U I}^{*}$, and $\left(g_{U I}, g_{S T C}\right)^{*}$ and the first best. These correspond to the theoretical labor input profiles of Figure 1, showing hours and employment as a function of profitability $x .^{26}$ Thick gray segments indicate the region of STC take-up.

Experiment $g_{U I}^{*}$ shows that optimal UI is somewhat above the calibrated level. ${ }^{27}$ The corresponding level of $\bar{n}$ is 0.968 , compared to 0.984 in the calibration. Thus the number of workers on layoff doubles. Hence layoffs respond quite strongly to $g_{U I}$, a point we return to below. Employment is below one at sufficiently low levels of profitability and increasing. Hours are constant over the profitability range with positive layoffs and increasing otherwise, as established in Part 1 of Proposition 3. In contrast, first-best employment is one irrespective of profitability, and first-best hours are increasing throughout.

Experiment $g_{S T C}^{*} \mid g_{U I}^{*}$ shows that introducing STC is optimal when UI is fixed at $g_{U I}^{*}$, and it establishes half of our first main result: under perfect PI, STC can substantially improve welfare, here by $0.3 \%$. The optimal level of $g_{S T C}$ is modest: the implied replacement rate for STC is $7.97 \%$, compared

\footnotetext{
${ }^{25} R E P R_{U I}$ is defined as the ratio between $g_{U I}$ and average consumption, expressed in percentage terms. Analogously, $R E P R_{S T C}$ is defined as the ratio between the maximal STC benefit $g_{S T C} \bar{h}$ and average consumption. Thus the two replacement rates coincide if $g_{S T C} \bar{h}=g_{U I}$.

${ }^{26}$ The $\mathrm{x}$-axis is scaled to the distribution of profitability shocks.

${ }^{27}$ Superficially this resembles Chetty's (2008) finding that the existing level of UI is somewhat below but close to optimal, yet the underlying trade-offs are different. In our setting UI distorts layoff decisions while worker moral hazard is absent. In contrast, Chetty's approach takes into account moral hazard but not distortions of layoff decisions.
} 

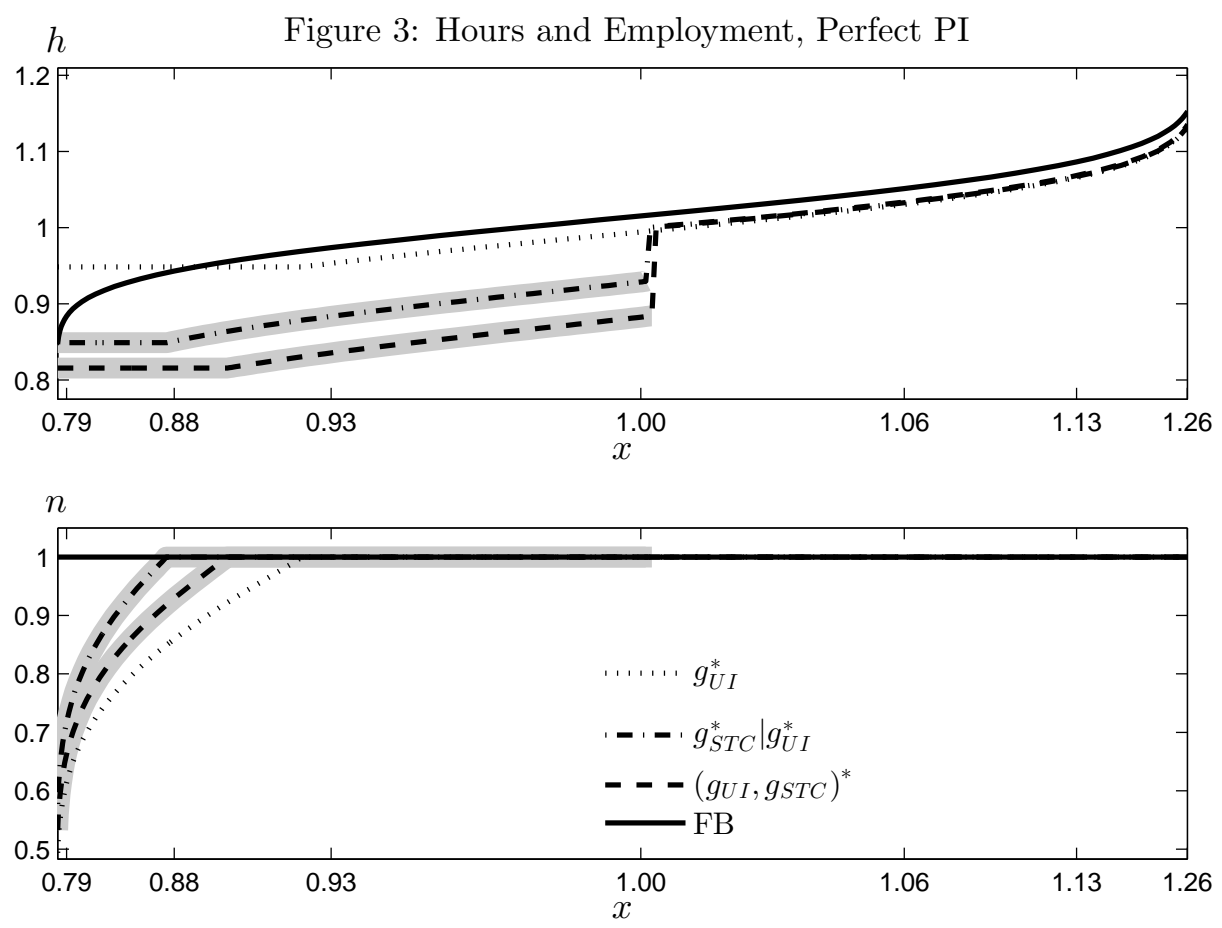

to $27.1 \%$ for UI. Nevertheless, this level of STC is quite effective, reducing layoffs by more than half. As discussed in Section 3.2.1, an increase in employment is not implied by our theoretical analysis, but occurs in all of our computational experiments. Hours per worker drop substantially, so that output is lower than in experiment $g_{U I}^{*}$, despite higher employment. Lower spending on UI outweighs spending on STC, and government outlays as a percentage of output are reduced from $2.19 \%$ under experiment $g_{U I}^{*}$ to $2 \%$. The labor input profiles for this experiment conform to Propositions 3 and 5 . The take-up threshold $x_{T}$ lies above the threshold $x_{0}^{N}$ at which the employment constraint becomes binding under no take-up. ${ }^{28}$ Thus some firms taking up STC would have retained all workers even in the absence of STC. For these firms STC distorts hours without the benefit of reducing layoffs. Employment is then continuous at the take-up threshold, while hours jump up. Throughout the take-up region, hours are strictly lower than in experiment $g_{U I}^{*}$ and employment is uniformly higher.

In experiment $g_{S T C}^{\max } \mid g_{U I}^{*}$, STC eliminates layoffs completely, but induces a very large decline in hours. Overall, this leads to a large welfare loss of $1.85 \%$ vis-à-vis experiment $g_{U I}^{*}$. Together with the preceding experiment, this establishes our second main result: Optimal STC is substantially less generous than UI, and introducing STC with equal generosity results in a large welfare loss in comparison to having no STC at all. In our model there is no natural reason for UI and STC to be equally generous. The optimal levels of UI and STC are determined by different trade-offs. Optimal UI balances the benefit of making

\footnotetext{
${ }^{28}$ Thresholds are not labeled in the figure, as it contains multiple experiments.
} 
Figure 4: Welfare Gains and Average Labor Inputs as Functions of $g_{S T C}$, Perfect PI

Welfare Gain Rel. to $g_{U I}^{*}$

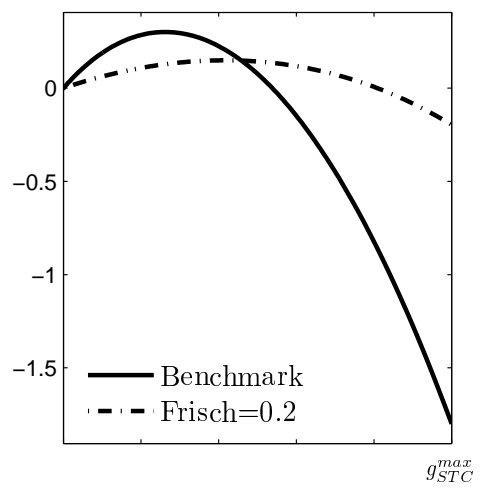

$g_{S T C}$ $\bar{n}$

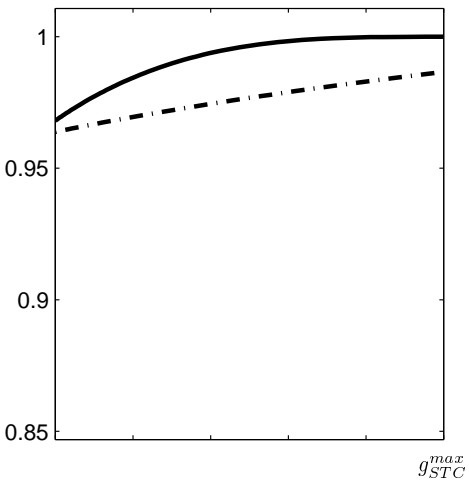

$g_{S T C}$ $\bar{h}$

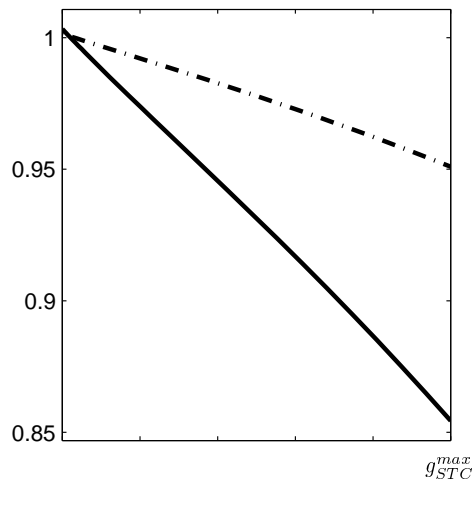

$g_{S T C}$

transfers to unattached workers against the cost of distorting the layoff decision of firms. Optimal STC balances mitigation of this distortion against the cost of distorting hours in firms that would abstain from layoffs even in the absence of STC. Figure 4 illustrates this result. The left panel plots the welfare gain as a function of $g_{S T C}$, with $g_{U I}$ fixed at $g_{U I}^{*}$ and $g_{S T C}$ varying up to $g_{S T C}^{\max }$. The middle and right panel show how average employment and hours vary with $g_{S T C}$. STC is quite effective in eliminating layoffs, in that employment already approaches the maximum level of one at intermediate levels of $g_{S T C}$. In contrast, average hours are falling linearly in $g_{S T C}$. When pushed beyond intermediate levels, few layoffs are left to eliminate, while the negative effect on average hours is undiminished. Thus the optimal STC is substantially below $g_{S T C}^{\max }$, and $g_{S T C}^{\max }$ yields a large welfare loss. For further intuition, the dashed-dotted lines in Figure 4 repeat the experiment with the model recalibrated to match a lower Frisch elasticity of 0.2. In this case firms are less willing to reduce hours in response to more generous STC. Thus layoffs are eliminated less quickly as $g_{S T C}$ increases. The optimal generosity of STC relative to UI is higher, yet the associated welfare gains are substantially lower. ${ }^{29}$

Experiment $\left(g_{U I}, g_{S T C}\right)^{*}$ shows that the optimal combination of UI and STC involves substantially more generous UI than under experiment $g_{U I}^{*}$ : the benefit level $g_{U I}$ increases by more than $8 \%$ (from 0.262 to 0.284 ), which corresponds to an increase in the replacement rate from $27.1 \%$ to $29.9 \%$. STC mitigates the distortions associated with UI, which in turn makes it optimal to offer more generous UI. Thereby the availability of STC improves insurance indirectly. As in experiment $g_{S T C}^{*} \mid g_{U I}^{*}$, STC is substantially less generous than UI. The welfare gain of moving from $g_{U I}^{*}$ to $\left(g_{U I}, g_{S T C}\right)^{*}$ is $0.53 \%$.

\footnotetext{
${ }^{29}$ This experiment indicates that, based on the forces at work in our model, it is not clear that countries in which the
} intensive margin plays a more important role should be expected to have a generosity of STC closer to that of UI: a higher effectiveness of STC can imply that less generous STC suffices to counteract layoffs. 
${ }_{h}$ Figure 5: Hours and Employment, Perfect PI: Minimum Hours Reduction
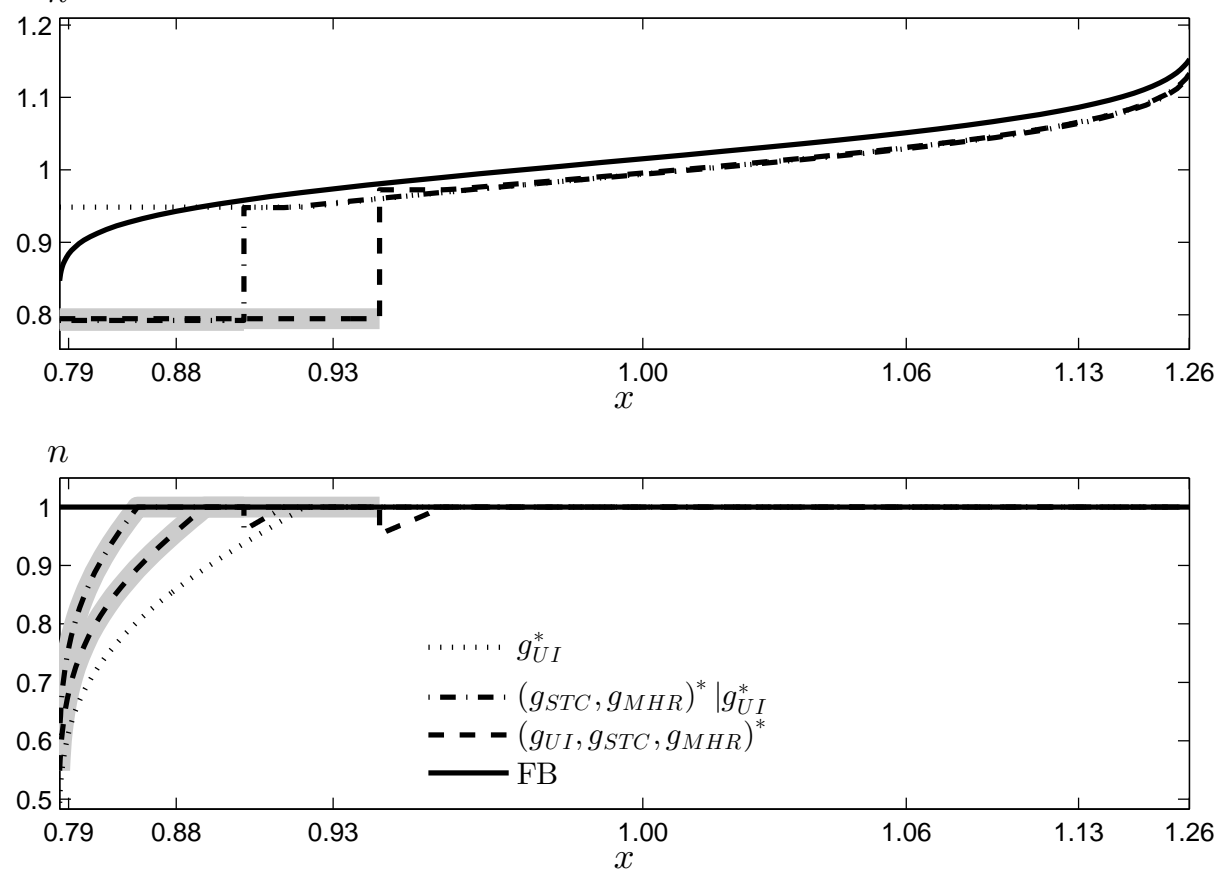

About half of this gain can be obtained by moving to $g_{S T C}^{*} \mid g_{U I}^{*}$, indicating that adjusting the level of UI is equally important to reap the full benefit of having STC as an additional instrument. Qualitatively the pattern of labor inputs across profitability in Figure 3 is very similar to the experiment $g_{S T C}^{*} \mid g_{U I}^{*}$. However, both hours and employment are lower since both UI and STC are more generous.

The next two experiments $\left(g_{S T C}, g_{M H R}\right)^{*} \mid g_{U I}^{*}$ and $\left(g_{U I}, g_{S T C}, g_{M H R}\right)^{*}$ allow the government to impose a minimum hours reduction (MHR). The optimal levels of $g_{M H R}$ shown in Table 3 are similar for the two experiments, at 0.81 and 0.82 , respectively, corresponding to minimum hours reductions of $19 \%$ and $18 \%$. This is close to the average of $20 \%$ observed across the 15 OECD countries with an MHR (Hijzen and Venn, 2011). The additional welfare gain is moderate: moving from $g_{U I}^{*}$ to $\left(g_{U I}, g_{S T C}, g_{M H R}\right)^{*}$ rather than $\left(g_{U I}, g_{S T C}\right)^{*}$ boosts the welfare gain by 0.08 percentage points, that is, by 15 percent of the welfare gain obtainable without MHR. As in the previous experiments without MHR, optimal STC is substantially less generous than UI. It becomes slightly less generous, as the MHR makes it possible to achieve the same reduction in layoffs with a lower level of $g_{S T C}$. The associated labor input profiles in Figure 5 show that the MHR binds throughout the take-up region. The take-up threshold is substantially lower than in the experiments without MHR, which is reflected in lower take-up rates in Table 3. Taxes are lower due to reduced expenditures on STC. Average hours and employment are higher.

Figure 6 illustrates how the MHR affects welfare and average labor input levels for given $g_{U I}$ and $g_{S T C}$. First consider the solid lines, which fix $g_{U I}$ and $g_{S T C}$ at $\left(g_{U I}, g_{S T C}\right)^{*}$. Lowering $g_{M H R}$ from a 
Figure 6: Welfare Gains and Average Labor Inputs as Functions of $g_{M H R}$, Perfect PI

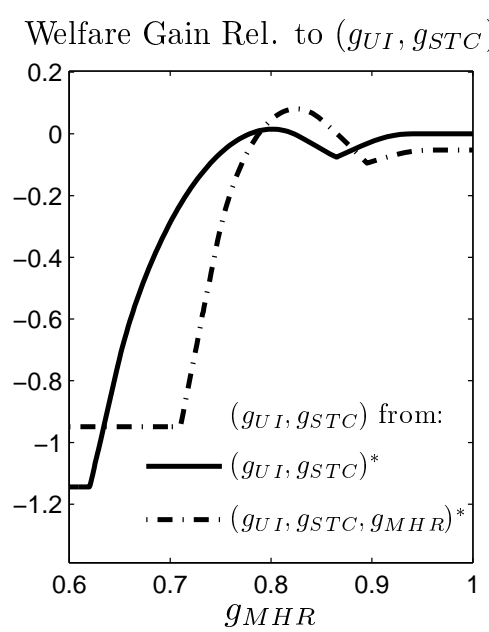

$\bar{n}$

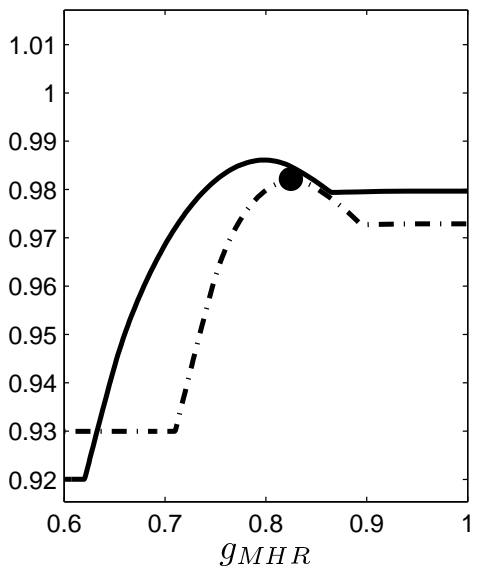

$\bar{h}$

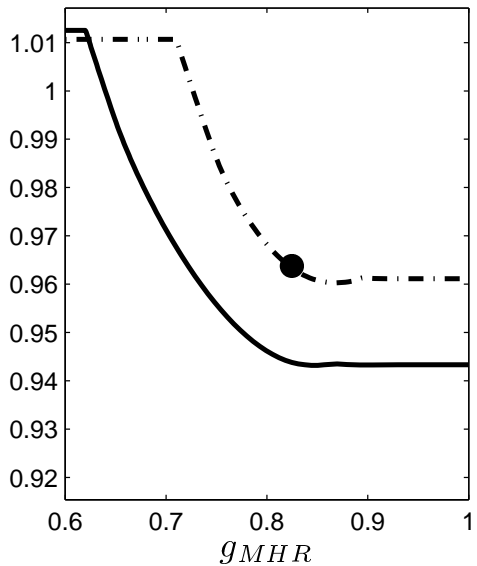

value of one at first as no effects as all firms taking up STC have hours strictly below one. As the MHR becomes binding with further reductions in $g_{M H R}$, some firms with $n=1$ choose to forgo STC. This is desirable as for these firms STC only distorts hours without a beneficial effect on employment. Yet other firms with $n=1$ reduce hours even further to meet the MHR. The combined effect on average employment and hours is very small. For welfare the negative effect dominates quantitatively. For further reductions in $g_{M H R}$, the MHR also binds for firms with $n<1$. In contrast to firms with $n=1$, for these firms imposing the MHR has the additional positive effect of reducing layoffs. Due to this effect, welfare now increases as $g_{M H R}$ is reduced further. The welfare-maximizing level of $g_{M H R}$ is attained in this region at $g_{M H R}=0.8$. This is very close to the optimal level in experiment $\left(g_{U I}, g_{S T C}, g_{M H R}\right)^{*}$ in which $g_{S T C}$ and $g_{U I}$ are reoptimized jointly with $g_{M H R}$. Yet welfare is only slightly higher than at $g_{M H R}=1$, by about $0.015 \%$ : welfare losses when the MHR binds only for firms with $n=1$ are barely overturned by welfare gains when it also binds for firms with $n<1$. Further reductions in $g_{M H R}$ lead to large negative effects due to inefficient hours reductions and because there are now more and more firms with $n<1$ that do not take up STC. Finally, there is a second flat region at very low levels of $g_{M H R}$ for which take-up is zero. Overall, with $g_{S T C}$ and $g_{U I}$ fixed at $\left(g_{U I}, g_{S T C}\right)^{*}$, the welfare-maximizing $g_{M H R}$ is substantially below one, yet the welfare gains are tiny. Thus optimizing $g_{U I}$ and $g_{S T C}$ jointly with $g_{M H R}$ is key for reaping the welfare gains found in the experiments above. This is illustrated by the dash-dotted lines in Figure 6, which fix $g_{S T C}$ and $g_{U I}$ at the reoptimized levels from experiment $\left(g_{U I}, g_{S T C}, g_{M H R}\right)^{*}$. Here the level of $g_{S T C}$ is substantially lower, which implies that at a given $g_{M H R}$, average employment is lower and average hours are higher. The MHR binds sooner for firms with $n<1$, hence the region with welfare gains from the MHR starts further to the right. The black dots in the plots 
Table 4: Policy Experiments: No PI

\begin{tabular}{lcccccc}
\hline \hline & Calibr. & $g_{U I}^{*}$ & $g_{S T C}^{*} \mid g_{U I}^{*}$ & $\bar{g}_{S T C} \mid g_{U I}^{*}$ & $\left(g_{U I}, g_{S T C}\right)^{*}$ & FB \\
\hline$g_{U I}$ & 0.247 & 0.25 & 0.25 & 0.25 & 0.25 & \\
$g_{S T C}$ & & & 0.00316 & 0.0758 & 0.00331 & \\
$g_{M H R}$ & & & 1 & 1 & 1 & \\
$\tau$ & 0.0157 & 0.017 & 0.017 & 0.018 & 0.0171 & \\
$R E P R_{U I}(\%)$ & 25 & 25.3 & 25.4 & 25.8 & 25.4 & \\
$R E P R_{S T C}(\%)$ & & & 0.321 & 7.6 & 0.336 & \\
STC Take-Up (\%) & & & 78.9 & 51.3 & 78.8 & \\
$\bar{n}$ & 0.984 & 0.98 & 0.98 & 0.984 & 0.98 & 1 \\
$\bar{h}$ & 1 & 1 & 0.999 & 0.969 & 0.999 & 1.02 \\
$\bar{y}$ & 0.996 & 0.994 & 0.992 & 0.975 & 0.992 & 1.02 \\
$\bar{c}$ & 0.984 & 0.982 & 0.981 & 0.963 & 0.981 & 0.982 \\
Welf. Rel. to $g_{U I}^{*}(\% c)$ & -0.012572 & & 0.00092858 & -0.076012 & 0.0009721 & 7.0041 \\
\hline \hline
\end{tabular}

for average labor inputs indicate the welfare-maximizing level of $g_{M H R}$, and show that it is possible to achieve both higher average employment and higher average hours than in the experiment $\left(g_{U I}, g_{S T C}\right)^{*}$.

A noteworthy feature of the calibrated model is that the level of unemployment is very sensitive to policy. The local semielasticities of unemployment with respect to the replacement rate are 15.6 and 10.4 at $g_{U I}^{*}$ and $g_{S T C}^{*} \mid g_{U I}^{*}$, respectively. In their empirical analysis Costain and Reiter (2008) estimate a semielasticity of 3 . This suggests that the model may be missing features that reduce the semielasticity. Interestingly, the model implies that STC can play an important role in reducing the semielasticity. Specifically, the impact of an increase in the replacement rate on unemployment is much weaker when STC is adjusted optimally. At $g_{S T C}^{*} \mid g_{U I}^{*}$, for example, the associated local semielasticity is 4.52 .

\subsection{No Private Insurance}

Lack of access to PI opens an additional channel through which STC can affect welfare, via a direct insurance effect. At first sight, the analysis in Section 4.2 may suggest that this effect is positive. In Figure 3 hours per worker are increasing in profitability (weakly so in the region with positive layoffs). Taking this as given, STC improves insurance by reallocating consumption to less profitable firms. However, the hours profile in Figure 3 is optimal when firms have access to perfect PI. As seen in Section 3, if firms lack access to PI, then the hours profile is declining over the profitability region in which firms engage in layoffs. This has important implications for the welfare effects of STC.

Table 4 reports the results of the policy experiments. The optimal level of $g_{S T C}$ in experiment $g_{S T C}^{*} \mid g_{U I}^{*}$ is positive but very close to zero at 0.003 , yielding a negligible welfare gain of 0.93 per million. This gain is imperceptible in the left panel of Figure 7, which plots welfare gains for levels of STC up to 
Figure 7: Welfare Gains, No PI
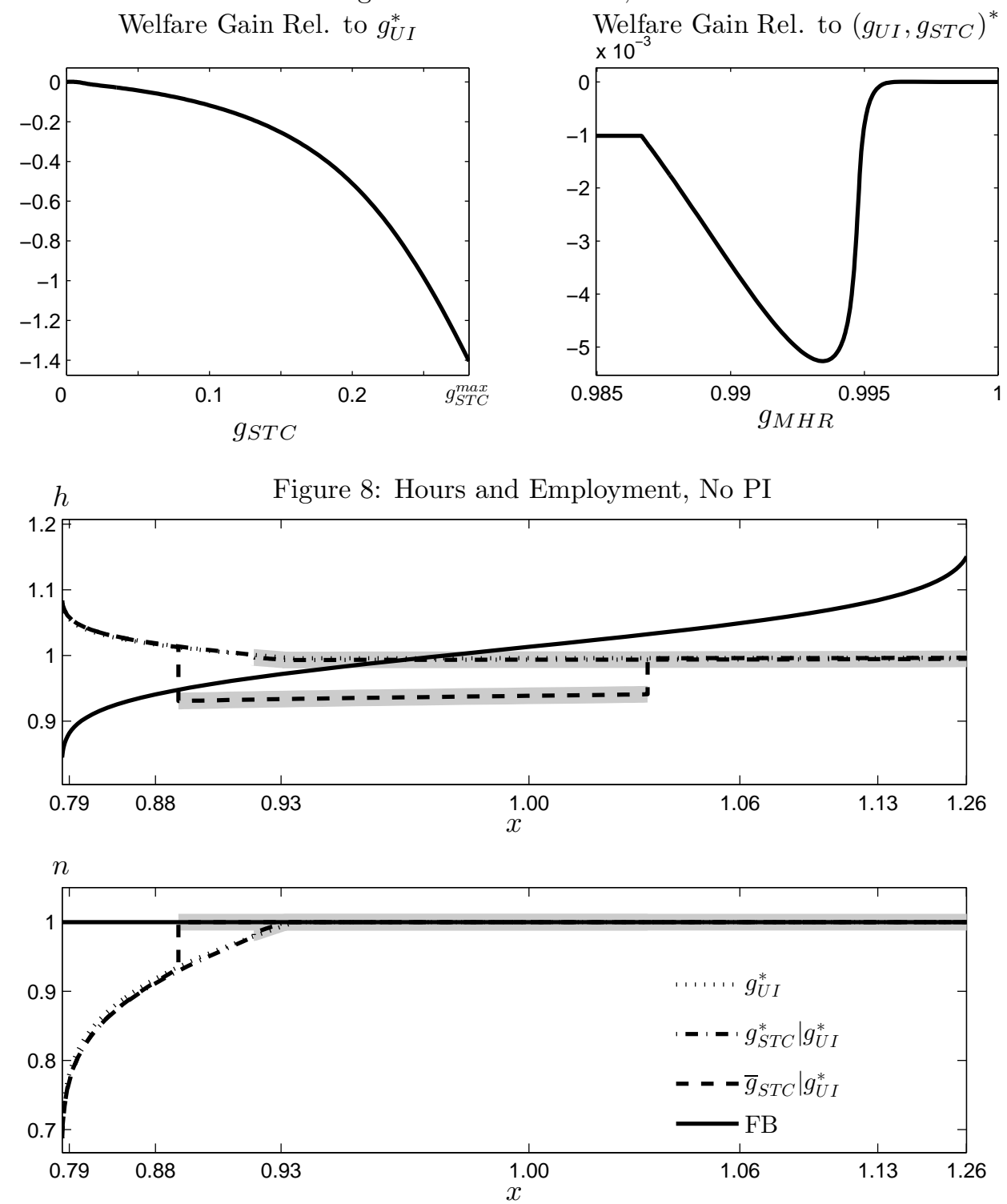

$g_{S T C}^{\max }=g_{U I}^{*} / \bar{h}$, given $g_{U I}^{*}$. This finding completes our first main result: STC delivers substantial welfare gains if firms have access to perfect PI, but fails to do so if firms have no access to PI.

Since the optimal level of STC in experiment $g_{S T C}^{*} \mid g_{U I}^{*}$ is so small, the availability of STC has a negligible impact on the optimal level of UI when both are optimized jointly in experiment $\left(g_{U I}, g_{S T C}\right)^{*}$.

Under perfect PI, a modest level of STC is optimal. To understand why STC of a similar magnitude is not optimal here, we conduct an additional experiment labeled $\bar{g}_{S T C} \mid g_{U I}^{*}$. Here $\bar{g}_{S T C}$ is chosen such that the ratio of STC to UI is the same as in the experiment $g_{S T C}^{*} \mid g_{U I}^{*}$ under perfect PI. Figure 8 is the counterpart of Figure 3, showing labor input profiles for this new experiment, together with the familiar experiments $g_{U I}^{*}$ and $g_{S T C}^{*} \mid g_{U I}^{*}$. Starting with experiment $g_{U I}^{*}$, the key difference vis-à-vis Figure 3 is that 
Figure 9: Change in Net Transfers Due to STC, No PI

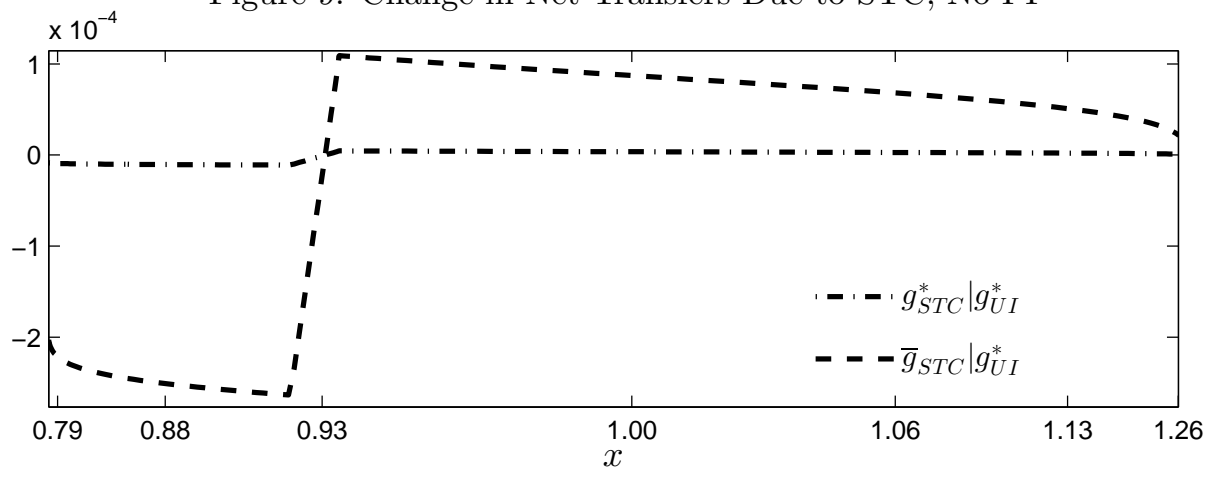

hours per worker are strictly decreasing rather than increasing over the profitability range with positive layoffs, in accordance with Proposition 4. Hours are strictly increasing over the region with $n=1$, in line with Proposition 4, yet quantitatively they are virtually flat. Recall from Section 3.2.2 that with perfect PI the strictly increasing pattern of hours over this region is due to a substitution effect. For the case of no PI, this substitution effect is counteracted by an income effect. With KPR preferences, the income effect would fully offset the substitution effect, were it not for the presence of the tax. Quantitatively the impact of the tax is small. Since the optimal level of STC in experiment $g_{S T C}^{*} \mid g_{U I}^{*}$ is very small, labor input profiles lie virtually on top of profiles from experiment $g_{U I}^{*}$. The thick gray segments indicate that take-up of STC occurs at high levels of profitability, distorting hours without any benefit in terms of reduced layoffs. The effects of STC are easier to see for the new experiment $\bar{g}_{S T C} \mid g_{U I}^{*}$. Here only firms with intermediate profitability take up STC, and for most of these firms STC merely distorts hours, without any reduction in layoffs. Firms with very low profitability, which account for most layoffs, forego STC in favor of high hours. Thereby the $V$-shaped pattern of hours severely weakens the positive welfare effect of STC observed in the case of perfect PI. Correspondingly, Table 4 shows little positive impact on employment, whereas hours and welfare decline significantly in this experiment.

The $V$-shaped pattern of hours also works against the ability of STC to improve welfare through the new effect arising in this scenario, namely the direct insurance effect. The most distressed firms forego STC, thus STC reallocates consumption away from this group. STC shifts consumptions in the right direction within the group of firms with $n=1$, since hours are strictly increasing over the corresponding profitability region. This effect is small, however, since hours are virtually flat over this region.

To quantify the overall direct insurance effect of STC, we fix labor input decisions at those from experiment $g_{U I}^{*}$ and calculate net government transfers induced by the levels of STC from experiment $g_{S T C}^{*} \mid g_{U I}^{*}$ in conjunction with a corresponding budget clearing tax $\tau$. Figure 9 plots the difference between the resulting net-transfer schedule and the net-transfer schedule from experiment $g_{U I}^{*}$. Clearly, 
net transfers worsen for the group of low profitability firms who do no take-up STC. Within the group of firms that take up STC, those with lower profitability gain more. The welfare effect of this change in net transfers is negative, and very small quantitatively at 0.79 per million. Of course this reflects in part that $g_{S T C}$ is very small. The direct insurance effect in experiment $\bar{g}_{S T C} \mid g_{U I}^{*}$ is $-0.002 \%$. This is negligible in comparison to the welfare gain of $0.3 \%$ induced by a similar STC level in the case of perfect PI. Thus the failure to substantially improve welfare is explained by the weakened ability to mitigate labor input distortions, rather than a negative direct insurance effect.

The finding of small direct insurance effects of STC does not mean that STC can be evaluated in a model with risk neutral firms, if firms do in fact lack access to PI. The degree of access to financial markets shapes how firms adjust labor inputs in response to shocks, and this matters for the ability of STC to affect labor input decisions. The experiment $\bar{g}_{S T C} \mid g_{U I}^{*}$ shows that the level of STC that is optimal if firms have access to perfect PI leads to a moderate welfare loss of 0.076 percent if firms lack access to private PI. Thus our model suggests that to the extent that the government can observe firm's access to PI, it is desirable to have different levels of STC for different groups of firms.

The option to combine STC with an MHR does not yield any additional welfare gains, that is, $g_{M H R}=1$ is optimal. Thus experiments $\left(g_{S T C}, g_{M H R}\right)^{*} \mid g_{U I}^{*}$ and $\left(g_{U I}, g_{S T C}, g_{M H R}\right)^{*}$ are omitted from Table 4. The right panel of Figure 7 is the counterpart of the corresponding panel of Figure 4, and illustrates that the introduction of an MHR does not improve welfare in the experiment $\left(g_{S T C}, g_{U I}\right)^{*}$. As in Figure 4 the impact of reducing $g_{M H R}$ is non-monotone. Once the MHR is low enough to bind, welfare first decreases and then increases, but never exceeds the level obtained for $g_{M H R}=1 .{ }^{30}$

\section{Intensive-Margin Technology}

So far we have focused on a specification of technology in which hours of different workers are perfectly substitutable. Welfare effects of STC may vary with features of technology, such as substitutability of hours. In this section we take a first step in analyzing the role of technology. We consider a specification that, in terms of the substitutability of hours of different workers, lies at the opposite end of the spectrum in that there is no substitutability at all. Producing firms then have $n=1$, and adjustment occurs via the intensive margin or complete shutdown. We refer to this as the intensive-margin case.

We find that our main results also hold for this specification. First, STC yields substantial welfare gains only in the case with perfect PI. In fact, optimal STC is zero under no PI. Second, optimal STC is substantially less generous than optimal UI, and equally generous STC results in a large welfare loss.

\footnotetext{
${ }^{30}$ The kink in Figure 7 is not present here, as all firms adopting STC have employment $n=1$.
} 
Table 5: Calibration: Intensive-Margin Case

\begin{tabular}{lccl}
\hline \hline Value & Perfect PI & No PI & Target \\
\hline$\sigma$ & 2 & 2 & \\
$\alpha$ & 0.67 & 0.67 & \\
$\sigma_{x}$ & 0.1 & 0.1 & \\
$\psi$ & 1.1 & 1.1 & Frisch elasticity 0.7 \\
$\eta$ & 0.663 & 0.661 & $\bar{h}=1$ (Normalization) \\
$v$ & 0.045 & 0.045 & Unattached workers 0.045 \\
$v_{0}, \% c, \% h$ & $0.712,29,41$ & $0.425,57,87$ & Temporary layoffs 0.015 \\
$g_{U I}$ & 0.246 & 0.245 & Replacement rate $25 \%$ \\
\hline \hline
\end{tabular}

Two main differences emerge vis-à-vis the standard technology. First, welfare gains of STC under perfect PI are smaller, because STC can reduce excessive layoffs caused by UI only via the relatively unresponsive shutdown margin. Second, STC now has a positive direct insurance effect in the no-PI scenario, although this effect is too small to make STC worthwhile.

BW provide a general specification that nests both the standard and the intensive margin technology: $l(n, h)$ is a function that combines employment and hours into a labor-input index, and output is given by $x f(l(n, h))$. The standard case is $l(n, h)=n h$. The intensive-margin case is

$$
l(n, h)= \begin{cases}h & \text { for } n=1 \\ 0 & \text { for } n<1\end{cases}
$$

The labor-input index is zero unless all attached workers have positive hours, hence adjusting hours per worker is the only possible response to a profitability shock, short of not producing. This and the standard case are the two ends of the spectrum of specifications exhibiting a property which BW refer to as Assumption $L$. It requires that technology is not biased against work sharing, in that reducing hours per worker while keeping total hours constant does not reduce output. ${ }^{31}$ Work sharing is neutral for the standard technology as output depends only on total hours. The intensive-margin case is most favorable to work sharing: reducing employment for given total hours results in a complete loss of output.

We do not need to revisit the theoretical analysis of Section 3 for this specification. The only change is that there is no longer a region in which employment lies strictly between zero and one. The analysis of the behavior of hours per worker when the employment constraint binds carries over directly. Thus we immediately turn to the sequence of computational experiments described in Section 4.

The calibration for the two scenarios of perfect and no PI is shown in Table 5. Parameters are

${ }^{31}$ Formally, the function $l(n, h)$ satisfies Assumption $L$ if $n_{2} h_{2}=n_{1} h_{1}$ and $n_{2}>n_{1}$ imply $l\left(n_{2}, h_{2}\right) \geq l\left(n_{1}, h_{1}\right)$. 
Table 6: Intensive-Margin Case, Perfect PI

\begin{tabular}{lcccccc}
\hline \hline & Calibr. & $g_{U I}^{*}$ & $g_{S T C}^{*} \mid g_{U I}^{*}$ & $g_{S T C}^{\max } \mid g_{U I}^{*}$ & $\left(g_{U I}, g_{S T C}\right)^{*}$ & FB \\
\hline$g_{U I}$ & 0.246 & 0.268 & 0.268 & 0.268 & 0.272 & \\
$g_{S T C}$ & & & 0.0516 & 0.315 & 0.0566 & \\
$g_{M H R}$ & & & 1 & 1 & 1 & \\
$\tau$ & 0.0157 & 0.0218 & 0.0221 & 0.0542 & 0.0236 & \\
$R E P R_{U I}(\%)$ & 25 & 27.6 & 27.8 & 30 & 28.3 & \\
$R E P R_{S T C}(\%)$ & & & 5.25 & 30 & 5.76 & \\
STC Take-Up (\%) & & & 49.6 & 48.8 & 49.5 & \\
$\bar{n}$ & 0.984 & 0.968 & 0.975 & 0.994 & 0.972 & 1 \\
$\bar{h}$ & 1 & 1 & 0.979 & 0.85 & 0.977 & 1.02 \\
$\bar{y}$ & 0.996 & 0.984 & 0.975 & 0.904 & 0.972 & 1.02 \\
$\bar{c}$ & 0.984 & 0.971 & 0.963 & 0.892 & 0.959 & 0.986 \\
Welf. Rel. to $g_{U I}^{*}(\% c)$ & -0.21582 & & 0.074817 & -2.2137 & 0.082714 & 4.138 \\
\hline \hline
\end{tabular}

essentially unchanged, with exception of the fixed-cost parameter $v_{0}$. Layoffs are much less attractive in the intensive-margin case, as they result in a complete loss of output. Matching the target for temporary layoffs then requires a substantially higher fixed cost of working positive hours. For the case of perfect PI, it amounts to a consumption-equivalent value of $28.8 \%$ as opposed to $6.63 \%$ for the standard case. Similarly, for the case of no PI, this cost increases from $11 \%$ to $57.5 \%$ in consumption equivalents. ${ }^{32}$

\subsection{Perfect Private Insurance}

Table 6 contains the results for the case of perfect PI. The left panel of Figure 10 shows welfare as a function of $g_{S T C}$ with UI fixed at $g_{U I}^{*}$. Introducing STC is optimal, yet comparison with Figure 4 shows that the ability of STC to improve welfare is more limited. The welfare-maximizing level is lower relative to $g_{U I}^{*}$, and the gain of $0.075 \%$ is relatively small. The reason is that STC is less effective in mitigating the distortion of layoffs caused by UI. The employment profiles in Figure 11 exhibit a shutdown region with $n=0$ for low profitability, immediately followed by an operating region with $n=1$. There is no intermediate region with employment strictly between zero and one. Thus STC cannot raise employment at the margin at a given level of $x$, affecting employment only by reducing shutdowns. In proportion to $g_{S T C}^{*}=0.052$, the increase in employment from 0.968 to 0.975 is small relative to the response under the standard technology. Meanwhile, the adverse effect of reducing hours for firms that would have chosen $n=1$ even in the absence of STC is about out as strong as for the standard technology. Experiment

\footnotetext{
32 By targeting aggregate temporary layoffs, we implicitly impose that all firms have the intensive-margin technology. If some sectors are closer to the standard case, while others are closer to the intensive-margin case, then one may expect more temporary layoffs in the former. It would be interesting to consider sector-specific calibrations in future research.
} 
Figure 10: Welfare Gains, Intensive-Margin Case, Perfect PI

Welfare Gain Rel. to $g_{U I}^{*}$

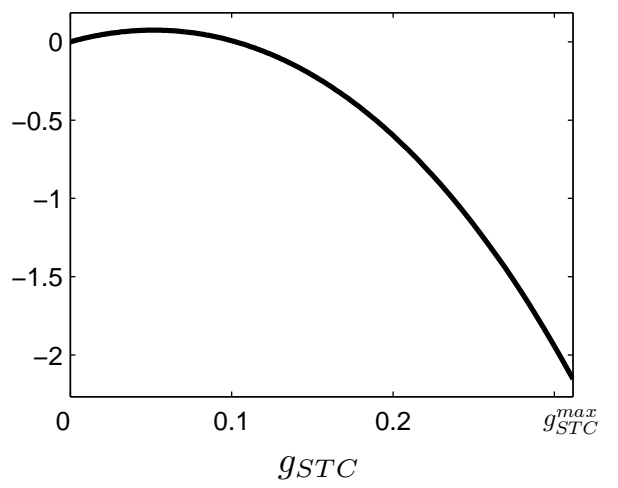

Welfare Gain Rel. to $\left(g_{U I}, g_{S T C}\right)^{*}$

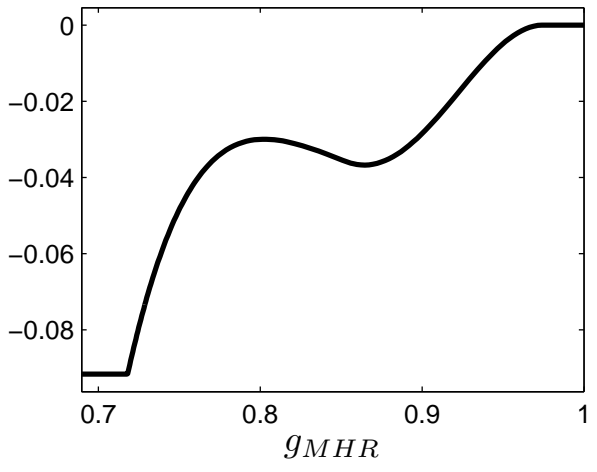

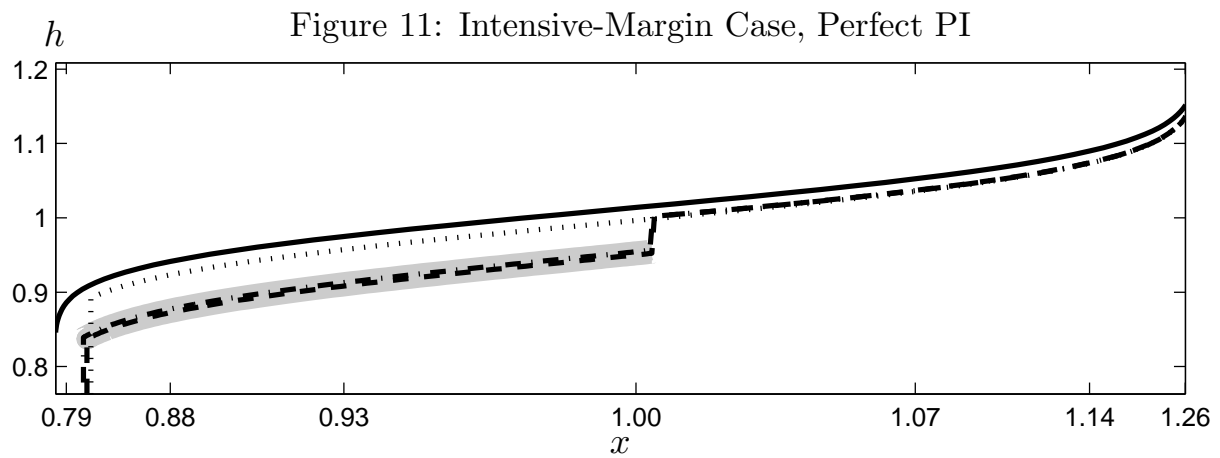

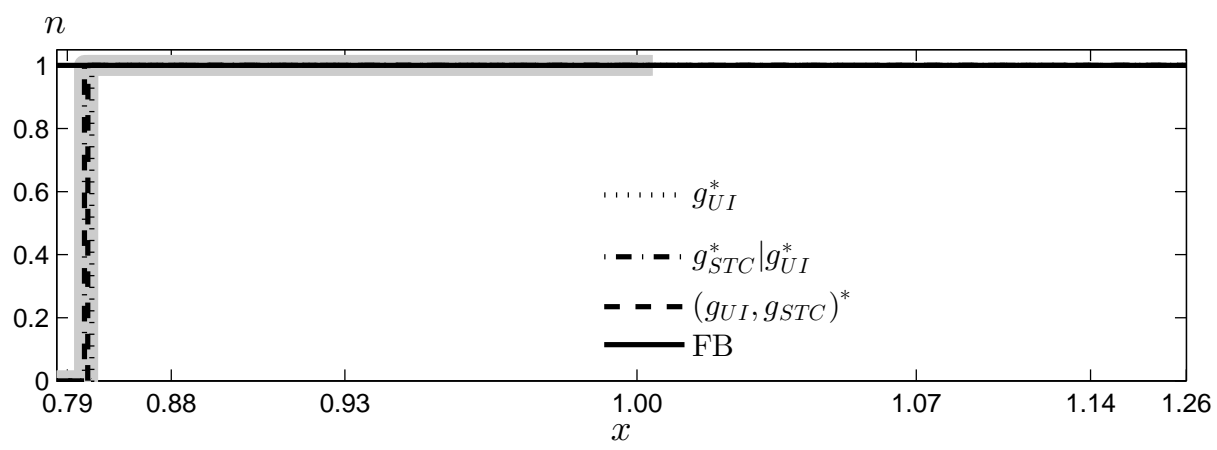

$g_{S T C}^{\max } \mid g_{U I}^{*}$ shows that as in the standard case, making STC as generous as UI results in a large welfare loss. In experiment $\left(g_{U I}, g_{S T C}\right)^{*}$, the availability of STC again improves insurance indirectly by allowing for more generous UI, but here the associated welfare gains are negligible.

Table 6 omits experiments involving $g_{M H R}$, because imposing an MHR is not optimal. This is illustrated in the right panel of Figure 10, which shows that an MHR cannot increase welfare further in experiment $\left(g_{U I}, g_{S T C}\right)^{*}$. As in Figure 4, at first there is a flat segment as $g_{M H R}$ is reduced below one because the MHR does not yet bind. As $g_{M H R}$ is reduced further, the usual trade-off arises as some firms forego STC and other firms reduce hours further to meet the MHR. The effect on welfare is non-monotone. In contrast to Figure 4, welfare always remains below the level at $g_{M H R}=1$. 
Table 7: Intensive-Margin Case, No PI

\begin{tabular}{lcccc}
\hline \hline & Calibr. & $g_{U I}^{*}$ & $\bar{g}_{S T C} \mid g_{U I}^{*}$ & $\mathrm{FB}$ \\
\hline$g_{U I}$ & 0.245 & 0.239 & 0.239 & \\
$g_{S T C}$ & & & 0.0723 & \\
$g_{M H R}$ & & & 1 & \\
$\tau$ & 0.0157 & 0.0131 & 0.0146 & \\
$R E P R_{U I}(\%)$ & 25 & 24.2 & 24.6 & \\
$R E P R_{S T C}(\%)$ & & & 7.24 & \\
STC Take-Up (\%) & & & 45.4 & \\
$\bar{n}$ & 0.984 & 0.993 & 0.992 & 0.94 \\
$\bar{h}$ & 1 & 1 & 0.973 & 1.03 \\
$\bar{y}$ & 0.993 & 1 & 0.983 & 0.978 \\
$\bar{c}$ & 0.981 & 0.989 & 0.972 & 0.953 \\
Welf. Rel. to $g_{U I}^{*}(\% c)$ & -0.063892 & & -0.13709 & 3.4684 \\
\hline \hline
\end{tabular}

\subsection{No Private Insurance}

STC has a direct insurance effect in the absence of PI. For the standard technology this effect is negative. This is driven by declining hours over the profitability region where some but not all workers are laid off. This region is absent in the intensive-margin case. All operating firms have $n=1$, and thus Proposition 4 implies a strictly increasing hours profile, which ensures a positive direct insurance effect of STC.

Introducing STC is not optimal, despite this positive insurance effect. This echoes the case of no PI under the standard technology, where optimal STC is very small. Here STC is less desirable, due to a relatively low optimal level of UI. In all preceding experiments, optimal UI exceeds its calibrated level, hence the associated level of layoffs exceeds the calibration target. In contrast, here $g_{U I}^{*}$ is below the calibrated level. At $0.7 \%$ the corresponding level of layoffs is far below the calibration target, leaving little room for STC to reduce layoffs. To illustrate what this means for the effects of STC, we again conduct experiment $\bar{g}_{S T C} \mid g_{U I}^{*}$, introducing a level of STC that is as generous as the optimal level under perfect PI. The results are displayed along with the experiment $g_{U I}^{*}$ and the first best in Table 7. Introducing STC even reduces employment slightly. The top panel of Figure 12 shows that STC still leads to a strong reduction of hours. Without a strong positive response of employment, UI payments cannot fall enough to offset the costs of STC. Thus the tax rate $\tau$ must increase, inducing the perverse employment effect. This emphasizes the importance of the magnitude of layoffs for the welfare benefits of STC.

The low level of $g_{U I}^{*}$, in turn, is due to a high marginal welfare loss from increasing the tax rate $\tau$. This is generated by the interaction between the intensive-margin technology and the lack of insurance, which puts firms with low profitability in an especially adverse position. Under the standard technology, firms can smoothly adjust to profitability shocks through layoffs. This smooth adjustment is not possible 

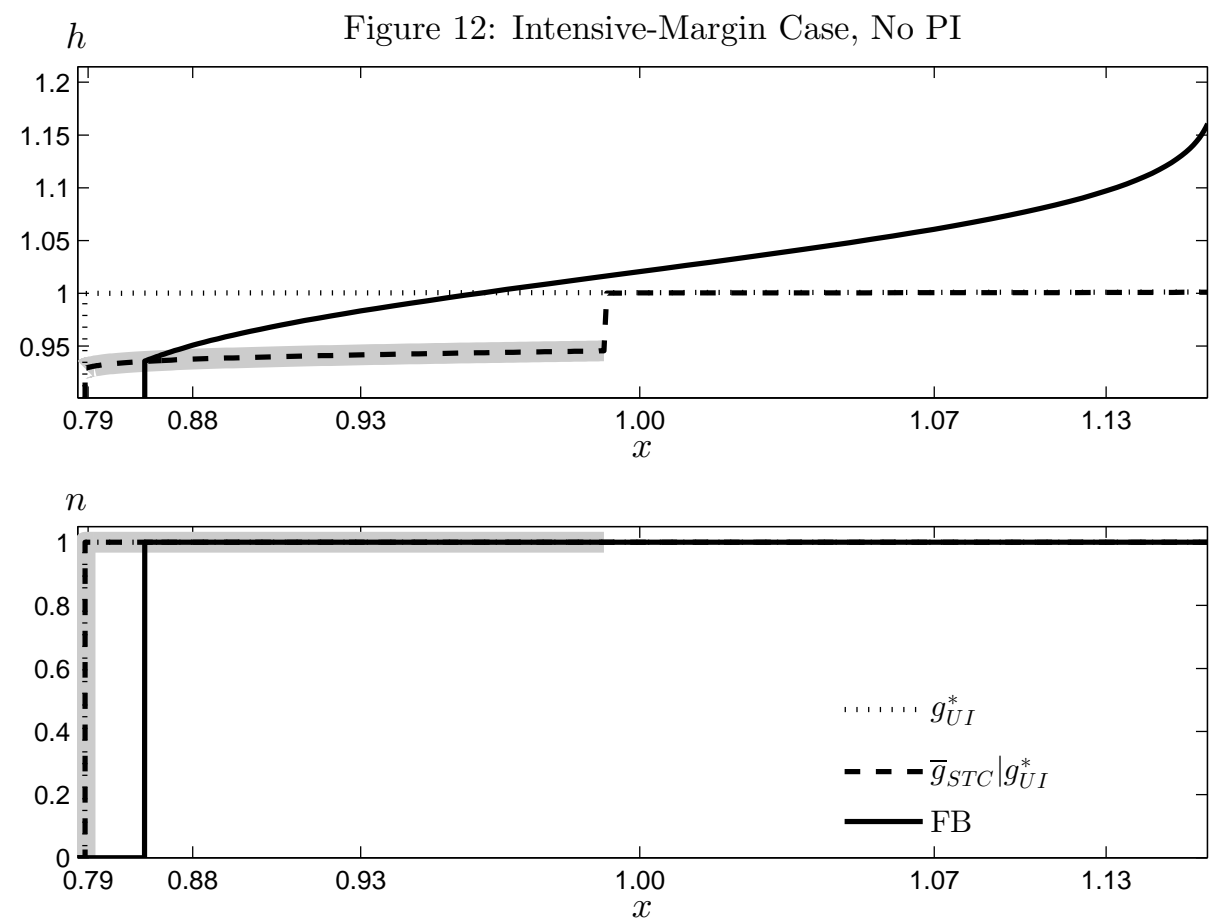

Figure 13: Intensive-Margin Case, Change in Net Transfers Due to STC, No PI

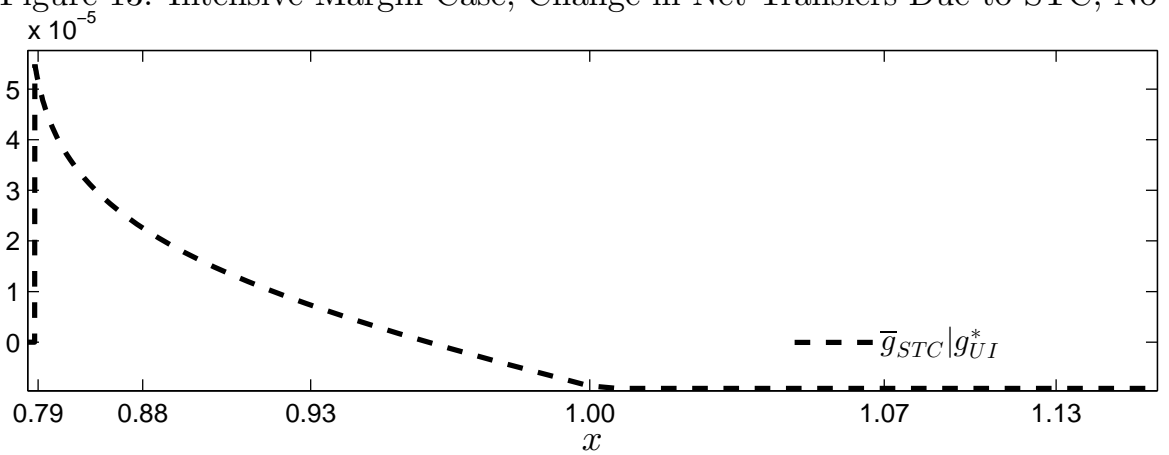

here. As a consequence, the average marginal utility from consumption across firms is substantially higher than in the standard case, making it very costly to raise revenue for financing UI.

While the direct insurance effect is positive, it is too small to matter. Figure 13 is constructed in the same way as Figure 9, and shows that the net transfers induced by STC in experiment $\bar{g}_{S T C} \mid g_{U I}^{*}$ shift consumption towards low profitability states. The magnitude of these transfers is very small, because the hours profile is virtually flat for the reason discussed in Section 4.3. Consequently, the direct insurance effect on welfare, computed as in Section 4.3, is very small at $0.00023 \%$. Overall, then, STC does not directly improve insurance in the settings we have studied in this paper. It only has the potential to do so in the absence of perfect PI. Yet the shape of the hours profile precludes substantial positive effects. 


\section{Conclusion}

We have studied welfare effects of short-time compensation (STC), departing from previous work by considering a setting in which unemployment insurance (UI) is socially optimal, and obtained two main results. First, STC can substantially improve welfare compared to a system that only relies on UI, but only when firms have access to private insurance (PI). Second, optimal STC is substantially less generous than UI even if firms have access to PI, and equally generous STC is worse than not offering STC.

In the course of this analysis, the paper also contributes a new comparative statics result to the implicit contracts literature: lacking access to private insurance, firms engaging in layoffs respond to a further decline in profitability by reducing employment and increasing hours per worker. This property is a key determinant of the welfare effects of STC and a testable implication of the model. Thus a natural next step is to investigate this implication empirically.

We see our analysis as groundwork for studying the welfare effects of STC in dynamic models of the labor market. As discussed in the introduction, dynamic models capturing all the features which make implicit contract models a natural choice for studying STC have not yet been developed. Given this, a potentially fruitful next step is to consider a variety of dynamic models, each retaining some of the features of the static model. The findings of the present paper can help to identify which models are likely to be interesting, as well has indicate potential pitfalls.

A relatively straightforward dynamic extension is a model in which firms face credit constraints and self-insure against fluctuations in profitability. Here one could maintain the simplification that attached agents are homogeneous, and assume that attachment is permanent. This setting would be especially interesting for revisiting the direct insurance effect of STC. In our static setting, the lack of private insurance affects low and high profitability levels symmetrically. This generates a flat hours profile across high profitability states without layoffs, as firms cannot save. The option to save would make STC less attractive for highly profitable firms and hours would rise more strongly in response to a temporary increase in profitability, potentially increasing the direct insurance effect of STC.

This extension still does not permit an evaluation of potential adverse effects of STC on worker reallocation. Introducing mobility of workers while maintaining incomplete markets may be intractable, however. Proceeding with complete markets and assuming exogenous UI would yield an interesting setting for studying the trade-off between STC's ability to mitigate distortions caused by UI, and potential reallocation effects. When interpreting results from this exercise, however, one should keep in mind that such a model may not capture well the STC take-up behavior of firms facing credit constraints. 


\section{A Proofs}

\section{Proposition 1}

Substituting the functions $c_{w}^{*}$ and $c_{b}^{*}$ into equation (18) and using functional form (1) yields

$$
\frac{\lambda^{-\frac{1-\sigma}{\sigma}}}{1-\sigma}-\frac{\lambda^{-\frac{1-\sigma}{\sigma}} v(h)^{\frac{1-\sigma}{\sigma}}}{1-\sigma}+\lambda^{-\frac{1-\sigma}{\sigma}} v(h)^{\frac{1-\sigma}{\sigma}} \frac{v^{\prime}(h) h}{v(h)}=\lambda^{-\frac{1-\sigma}{\sigma}}\left[1-v(h)^{\frac{1-\sigma}{\sigma}}\right]-\lambda\left[g_{U I}-\bar{h} T \cdot g_{S T C}\right] .
$$

Dividing both sides by $\lambda^{-\frac{1-\sigma}{\sigma}}$ and rearranging terms, we obtain

$$
\frac{\sigma}{1-\sigma}+v(h)^{\frac{1-2 \sigma}{\sigma}}\left[v^{\prime}(h) h-\frac{\sigma}{1-\sigma} v(h)\right]+\lambda^{\frac{1}{\sigma}}\left[g_{U I}-\bar{h} T \cdot g_{S T C}\right]=0
$$

Evaluating the left-hand side (LHS) at $h=0$ gives

$$
\frac{\sigma}{1-\sigma}\left(1-v_{0}^{\frac{1-\sigma}{\sigma}}\right)+\lambda^{\frac{1}{\sigma}}\left[g_{U I}-\bar{h} T \cdot g_{S T C}\right]>0
$$

This term captures the fixed cost of employing an additional worker. The first summand reflects the utility fixed cost, and is strictly positive since $v_{0} \in(0,1)$. The second term reflects the fixed cost in terms of the consumption good, induced by policy. It is nonnegative since $\bar{h} g_{S T C} \leq g_{U I}$. Let

$$
\tilde{V}(h) \equiv v(h)^{\frac{1-2 \sigma}{\sigma}}\left[v^{\prime}(h) h-\frac{\sigma}{1-\sigma} v(h)\right]
$$

Straightforward differentiation yields $\tilde{V}^{\prime}(h)=-h V^{\prime}(h)$, where $V(h)$ is defined in Section 2. As $V^{\prime}(h)>$ 0 , the LHS of equation (21) is strictly decreasing in $h$. Furthermore, $\lim _{h \rightarrow h_{\max }} \tilde{V}(h)=-\infty$ since $\lim _{h \rightarrow h_{\max }} V(h)=\infty$. Thus the LHS of equation (21) converges to $-\infty$ as $h$ converges to $h_{\max }$. Hence equation (21) has a unique solution in $\left(0, h_{\max }\right)$. If $g_{S T C}>0$, then the LHS of equation (21) is strictly decreasing in $T$, and thus the solution for hours is strictly decreasing in $T$. If $g_{U I}-\bar{h} T \cdot g_{S T C}>0$, then the LHS of equation (21) is strictly increasing in $\lambda$, hence the solution for hours is strictly increasing in $\lambda$. If $g_{U I}-\bar{h} T \cdot g_{S T C}=0$, then the solution for hours is independent of $\lambda$.

As discussed in the text, the result that hours are increasing in $\lambda$ if $g_{U I}-\bar{h} T \cdot g_{S T C}>0$ and independent of $\lambda$ if $g_{U I}-\bar{h} T \cdot g_{S T C}=0$ also holds for other common specifications of utility besides KPR. If preferences are additively separable, that is, $u(c, h)=\frac{c^{1-\sigma}}{1-\sigma}+v(h)$, then equation (18) becomes

$$
v^{\prime}(h) h-[v(h)-v(0)]+\lambda\left[g_{U I}-\bar{h} T \cdot g_{S T C}\right]=0 .
$$


Once again hours are strictly increasing in $\lambda$ if $g_{U I}-\bar{h} T \cdot g_{S T C}>0$ and independent of $\lambda$ otherwise. If utility takes the GHH form $u(c, h)=\frac{(c+v(h))^{1-\sigma}-1}{1-\sigma}$, then equation (18) becomes

$$
\lambda v^{\prime}(h) h-\lambda[v(h)-v(0)]+\lambda\left[g_{U I}-\bar{h} T \cdot g_{S T C}\right]=0 .
$$

In this case hours are independent of $\lambda$ for any value of $g_{U I}-\bar{h} T \cdot g_{S T C}$.

\section{Proposition 2}

As $V(h)$ is strictly increasing on $\left(0, h_{\max }\right), \lim _{h \rightarrow 0} V(h)$ is finite. Since $\lim _{h \rightarrow 0} f^{\prime}(h)=+\infty$, the righthand side (RHS) of equation (19) strictly exceeds the left-hand side (LHS) as $h$ converges to 0 . Since $\lim _{h \rightarrow h_{\text {max }}} V(h)=+\infty$ while $f^{\prime}\left(h_{\max }\right)$ is finite, the LHS of equation (19) strictly exceeds the RHS as $h$ converges to $h_{\max }$. Since $V$ is strictly increasing while $f^{\prime}$ is strictly decreasing, equation (19) has a unique solution in $\left(0, h_{\max }\right)$. The RHS is strictly increasing in $x$, hence the solution is strictly increasing in $x$. Suppose that the solution does not converge to $h_{\max }$ as $x$ converges to infinity. Then the LHS converges to a finite value while the RHS converges to infinity as $x$ converges to infinity, a contradiction. If $g_{S T C}>0$, then the RHS is strictly decreasing in $T$, hence the solution is strictly decreasing in $T$. Finally, note that at the solution the term in square brackets on the RHS is strictly positive. Thus the RHS is strictly increasing in $\lambda$, which implies that the solution for hours is strictly increasing in $\lambda$.

\section{Proposition 3}

We start with preliminary steps that apply to both $T=0$ and $T=1$. Let $\tilde{h}^{T}(\lambda)$ denote the level of hours that solves equation (18), which is well defined according to Proposition 1 . Let $\tilde{h}_{N}^{T}(x, \lambda)$ denote the level of hours that solves equation (19), which is well defined according to Proposition 2.

With $\chi=0$, first-order condition (16) implies that $\lambda(x)$ does not vary with $x$ at the solution. Let $\lambda^{*}$ denote its constant value. Substituting the functions $c_{w}^{*}$ and $c_{b}^{*}$ into equation (14), we can solve explicitly for the value that employment would have to take if (5) is slack:

$$
\begin{aligned}
\hat{n}^{T}(x, h) & \equiv\left(f^{\prime}\right)^{-1}\left(\frac { 1 } { x h } \left\{\frac{1}{\lambda^{*}}\left[u\left(c_{b}^{*}\left(\lambda^{*}\right), 0\right)-u\left(c_{w}^{*}\left(\lambda^{*}, h\right), h\right)\right]\right.\right. \\
& \left.\left.+g_{U I}-\bar{h} T \cdot g_{S T C}+\left(\tau+T \cdot g_{S T C}\right) h+c_{w}^{*}\left(\lambda^{*}, h\right)-c_{b}^{*}\left(\lambda^{*}\right)\right\}\right) \cdot \frac{1}{h} .
\end{aligned}
$$

As a function of $x$ for given $h, \hat{n}^{T}(x, h)$ is strictly increasing, and the Inada conditions on $f^{\prime}$ imply that $\hat{n}^{T}(x, h)$ converges to infinity as $x \rightarrow \infty$, and converges to zero as $x \rightarrow 0$. Thus there exists a unique threshold $\hat{x}_{N}^{T}(h)$ such that $\hat{n}^{T}\left(\hat{x}_{N}^{T}(h), h\right)=1$. Next we prove the parts of the proposition. 
1. Across levels of $x$ with a slack (5), hours are constant at $h^{0}(x)=\tilde{h}^{0}\left(\lambda^{*}\right)$, while employment is $n^{0}(x)=\hat{n}^{0}\left(x, \tilde{h}^{0}\left(\lambda^{*}\right)\right)$ and thus strictly increasing in $x$. Hence (5) becomes binding at $x_{N}^{0} \equiv$ $\hat{x}_{N}^{0}\left(\tilde{h}^{0}\left(\lambda^{*}\right)\right)$, thus it is slack on $\left(0, x_{N}^{0}\right)$ and binding on $\left(x_{N}^{0},+\infty\right)$. On the latter interval, hours are $h^{0}(x)=\tilde{h}_{N}^{0}\left(x, \lambda^{*}\right)$ and thus strictly increasing.

2. The proof is similar to that for Part 1, except (6) may bind. Let $\tilde{h}_{M H R}^{1} \equiv \min \left[\tilde{h}^{1}\left(\lambda^{*}\right), g_{M H R} \bar{h}\right]$. Then, across levels of $x$ with a slack (5), hours are constant at $h^{1}(x)=\tilde{h}_{M H R}^{1}$, while employment is $n^{1}(x)=\hat{n}^{1}\left(x, \tilde{h}_{M H R}^{1}\right)$ and thus strictly increases in $x$. Hence (5) becomes binding at $x_{N}^{1} \equiv \hat{x}_{N}^{1}\left(\tilde{h}_{M H R}^{1}\right)$. Thus it is slack on $\left(0, x_{N}^{1}\right)$ and binds on $\left(x_{N}^{1},+\infty\right)$. On the latter interval, hours are $h^{1}(x)=\min \left[\tilde{h}_{N}^{1}\left(x, \lambda^{*}\right), g_{M H R} \bar{h}\right]$. If $\tilde{h}_{N}^{1}\left(x_{N}^{1}, \lambda^{*}\right) \geq g_{M H R} \bar{h}$, set $x_{M H R}^{1}=x_{N}^{1}$. If $\lim _{x \rightarrow \infty} \tilde{h}_{N}^{1}\left(x, \lambda^{*}\right) \leq g_{M H R} \bar{h}$, set $x_{M H R}^{1}=+\infty$. Otherwise, set $x_{M H R}^{1}$ to the unique value of $x$ satisfying $\tilde{h}_{N}^{1}\left(x, \lambda^{*}\right)=g_{M H R} \bar{h}$. With this definition, hours $h^{1}(x)$ are strictly increasing on $\left(x_{N}^{1}, x_{M H R}^{1}\right)$, and constant at $g_{M H R} \bar{h}$ on $\left(x_{M H R}^{1},+\infty\right)$.

3. There are four cases. First, suppose $x \leq \min \left[x_{N}^{0}, x_{N}^{1}\right]$. Then $h^{0}(x)=\tilde{h}^{0}\left(\lambda^{*}\right)$ and $h^{1}(x)=$ $\min \left[\tilde{h}^{1}\left(\lambda^{*}\right), g_{M H R} \bar{h}\right]$. The desired result follows immediately from Proposition 1, which implies $\tilde{h}^{1}\left(\lambda^{*}\right)<\tilde{h}^{0}\left(\lambda^{*}\right)$. Second, consider $x \geq \max \left[x_{N}^{0}, x_{N}^{1}\right]$. Then $h^{0}(x)=\tilde{h}_{N}^{0}\left(x, \lambda^{*}\right)$ and $h^{1}(x)=$ $\min \left[\tilde{h}_{N}^{1}\left(x, \lambda^{*}\right), g_{M H R} \bar{h}\right]$. The desired result follows immediately from Proposition 2, which implies $\tilde{h}_{N}^{1}\left(x, \lambda^{*}\right)<\tilde{h}_{N}^{0}\left(x, \lambda^{*}\right)$. Third, suppose $x_{N}^{1}<x_{N}^{0}$ and consider $x \in\left[x_{N}^{1}, x_{N}^{0}\right]$. Now $h^{0}(x)=\tilde{h}^{0}\left(\lambda^{*}\right)$ and $h^{1}(x)=\min \left[\tilde{h}_{N}^{1}\left(x, \lambda^{*}\right), g_{M H R} \bar{h}\right]$. The desired result follows from

$$
\tilde{h}_{N}^{1}\left(x, \lambda^{*}\right) \leq \tilde{h}_{N}^{1}\left(x_{N}^{0}, \lambda^{*}\right)<\tilde{h}_{N}^{0}\left(x_{N}^{0}, \lambda^{*}\right)=\tilde{h}^{0}\left(\lambda^{*}\right)
$$

where the first inequality uses that $\tilde{h}_{N}^{1}\left(x, \lambda^{*}\right)$ is increasing in $x$, the second uses Proposition 2, and the final equality uses the definition of $x_{N}^{0}$. Fourth, suppose $x_{N}^{0}<x_{N}^{1}$ and consider $x \in\left[x_{N}^{0}, x_{N}^{1}\right]$. Here $h^{0}(x)=\tilde{h}_{N}^{0}\left(x, \lambda^{*}\right)$ and $h^{1}(x)=\min \left[\tilde{h}^{1}\left(\lambda^{*}\right), g_{M H R} \bar{h}\right]$. The desired result follows from

$$
\tilde{h}_{N}^{0}\left(x, \lambda^{*}\right) \geq \tilde{h}_{N}^{0}\left(x_{N}^{0}, \lambda^{*}\right)=\tilde{h}^{0}\left(\lambda^{*}\right)>\tilde{h}^{1}\left(\lambda^{*}\right)
$$

where the first inequality uses that $\tilde{h}_{N}^{0}\left(x, \lambda^{*}\right)$ is strictly increasing in $x$, the equality uses the definition of $x_{N}^{0}$, and the second inequality uses Proposition 1.

\section{Proposition 4}

We start with preliminary steps that apply to both $T=0$ and $T=1$. First, we analyze the comparative statics of hours and employment with respect to $x$ in a relaxed problem without constraints (5) and (6). 
Let $\tilde{n}^{T}(x)$ and $\tilde{h}^{T}(x)$ denote the optimal levels of employment and hours in this problem. Since $\chi=1$, constraint (8) is irrelevant, thus there is no interdependence of the optimization problem across levels of $x$, so we can solve it separately for each level of $x$. Only constraints (4) and (7) remain. We reduce the problem to an unconstrained one of choosing hours and employment. To do so, substitute the functions $c_{w}^{*}$ and $c_{b}^{*}$ along with $\iota=0$ into the budget constraint. Solving the resulting equation for $\lambda^{-\frac{1-\sigma}{\sigma}}$ yields

$$
\lambda^{-\frac{1-\sigma}{\sigma}}=\left[\frac{x f(n h)+(1-n) g_{U I}+n(\bar{h}-h) T \cdot g_{S T C}-\tau n h}{n v(h)^{\frac{1-\sigma}{\sigma}}+(1-n)}\right]^{1-\sigma} .
$$

Substituting the functions $c_{w}^{*}$ and $c_{b}^{*}$ along with $\iota=0$ into the objective yields

$$
n u\left(c_{w}, h\right)+(1-n) u\left(c_{b}, 0\right)=\frac{1}{1-\sigma} \lambda^{-\frac{1-\sigma}{\sigma}}\left[\left(n v(h)^{\frac{1-\sigma}{\sigma}}+(1-n)\right]-\frac{1}{1-\sigma} .\right.
$$

Using equation (23) to replace $\lambda^{-\frac{1-\sigma}{\sigma}}$ and dropping the constant $-\frac{1}{1-\sigma}$, the objective can be written as

$$
\frac{1}{1-\sigma}\left[x f(n h)+(1-n) g_{U I}+n(\bar{h}-h) T \cdot g_{S T C}-\tau n h\right]^{1-\sigma}\left[n v(h)^{\frac{1-\sigma}{\sigma}}+(1-n)\right]^{\sigma} .
$$

The optimal labor input levels $\tilde{n}^{T}(x)$ and $\tilde{h}^{T}(x)$ must maximize this objective. Since $\sigma>1$, this is equivalent to maximizing $G(n, h, x) \equiv \log [\Omega(n, h, x)]-\frac{1}{\psi} \log [\Gamma(n, h)]$ where $\psi \equiv \frac{\sigma-1}{\sigma} \in(0,1)$ and

$$
\begin{aligned}
\Omega(n, h, x) & \equiv x f(n h)+(1-n) g_{U I}+n(\bar{h}-h) T \cdot g_{S T C}-\tau n h, \\
\Gamma(n, h) & \equiv n v(h)^{-\psi}+(1-n) .
\end{aligned}
$$

To simplify notation, we suppress arguments of functions in what follows. The functions $\Omega$ and $\Gamma$ satisfy

$$
\Omega_{n h}=-x\left|f^{\prime \prime}\right| n h+\frac{1}{n} \Omega_{h} \quad \text { and } \quad \Gamma_{h h}=-n \psi v^{-(2+\psi)}\left[v v^{\prime \prime}-(1+\psi)\left(v^{\prime}\right)^{2}\right]>0 .
$$

The first-order conditions are

$$
G_{n}=\Omega^{-1} \Omega_{n}-(\psi \Gamma)^{-1} \Gamma_{n}=0 \quad \text { and } \quad G_{h}=\Omega^{-1} \Omega_{h}-(\psi \Gamma)^{-1} \Gamma_{h}=0
$$

From now on, all second derivatives are evaluated at the solution to these first-order conditions. Using that $\Omega_{x}=f, \Omega_{n x}=f^{\prime} h$, and $\Omega_{h x}=f^{\prime} n$, the second derivatives involving $x$ are

$$
G_{n x}=\Omega^{-2}\left(f^{\prime} h \Omega-\Omega_{n} f\right) \quad \text { and } \quad G_{h x}=\Omega^{-2}\left(f^{\prime} n \Omega-\Omega_{h} f\right) .
$$


Using that $\Omega_{n n}=-x\left|f^{\prime \prime}\right| h^{2}, \Omega_{h h}=-x\left|f^{\prime \prime}\right| n^{2}$, and equation (24), second derivatives for labor inputs are

$$
\begin{aligned}
& G_{n n}=-\Omega^{-2}\left(x\left|f^{\prime \prime}\right| h^{2} \Omega+\left(\Omega_{n}\right)^{2}\right)-\psi^{-1} \Gamma^{-2}\left(\Gamma_{n n} \Gamma-\Gamma_{n}^{2}\right) \\
& G_{h h}=-\Omega^{-2}\left(x\left|f^{\prime \prime}\right| n^{2} \Omega+\left(\Omega_{h}\right)^{2}\right)-\psi^{-1} \Gamma^{-2}\left(\Gamma_{h h} \Gamma-\Gamma_{h}^{2}\right), \\
& G_{n h}=-\Omega^{-2}\left(x\left|f^{\prime \prime}\right| n h \Omega-n^{-1} \Omega_{h} \Omega+\Omega_{n} \Omega_{h}\right)-\psi^{-1} \Gamma^{-2}\left(\Gamma_{n h} \Gamma-\Gamma_{n} \Gamma_{h}\right) .
\end{aligned}
$$

Using equation (24), $\Gamma_{n h}=n^{-1} \Gamma_{h}, \Gamma_{n n}=0$, and the first-order conditions, they can be written as

$$
\begin{aligned}
& G_{n n}=-\Omega^{-2}\left(x\left|f^{\prime \prime}\right| h^{2} \Omega+(1-\psi)\left(\Omega_{n}\right)^{2}\right), \\
& G_{h h}=-\Omega^{-2}\left(x\left|f^{\prime \prime}\right| n^{2} \Omega+(1-\psi)\left(\Omega_{h}\right)^{2}\right)-(\psi \Gamma)^{-1} \Gamma_{h h}, \\
& G_{n h}=-\Omega^{-2}\left(x\left|f^{\prime \prime}\right| n h \Omega+(1-\psi) \Omega_{n} \Omega_{h}\right) .
\end{aligned}
$$

The sign of $\frac{d}{d x} \tilde{h}^{T}(x)$ equals the sign of $-G_{n n} G_{h x}+G_{n h} G_{n x}$. Dropping the denominator $\Omega^{2}$, which appears in all four second derivatives involved in this expression, we see that it has the same sign as

$$
\left(x\left|f^{\prime \prime}\right| h^{2} \Omega+(1-\psi)\left(\Omega_{n}\right)^{2}\right)\left(f^{\prime} n \Omega-\Omega_{h} f\right)-\left(x\left|f^{\prime \prime}\right| n h \Omega+(1-\psi) \Omega_{n} \Omega_{h}\right)\left(f^{\prime} h \Omega-\Omega_{n} f\right) .
$$

Exploiting cancelations, this reduces to

$$
-\left[x\left|f^{\prime \prime}\right| h \Omega f+(1-\psi) \Omega \Omega_{n} f^{\prime}\right] \cdot\left[h \Omega_{h}-n \Omega_{n}\right] .
$$

Since, from the definition of $\Omega$,

$$
h \Omega_{h}-n \Omega_{n}=n\left[g_{U I}-\bar{h} T \cdot g_{S T C}\right]
$$

the expression in equation (26) is strictly negative if $g_{U I}-\bar{h} T \cdot g_{S T C}>0$ and zero if $g_{U I}-\bar{h} T \cdot g_{S T C}=0$.

The sign of $\frac{d}{d x} \tilde{n}^{T}(x)$ equals the sign of $-G_{h h} G_{n x}+G_{n h} G_{h x}$, and thus the sign of

$$
\left(x\left|f^{\prime \prime}\right| n^{2} \Omega+(1-\psi)\left(\Omega_{h}\right)^{2}\right)\left(f^{\prime} h \Omega-\Omega_{n} f\right)-\left(x\left|f^{\prime \prime}\right| n h \Omega+(1-\psi) \Omega_{n} \Omega_{h}\right)\left(f^{\prime} n \Omega-\Omega_{h} f\right)+\frac{\Omega^{4}}{\psi} \frac{\Gamma_{h h}}{\Gamma} G_{n x} .
$$

Except for the last term, this is symmetric to (25) with $n$ and $h$ switched. Thus it simplifies to

$$
\left[x\left|f^{\prime \prime}\right| n \Omega f+(1-\psi) \Omega \Omega_{h} f^{\prime}\right] \cdot\left[h \Omega_{h}-n \Omega_{n}\right]+(\psi \Gamma)^{-1} \Omega^{4} \Gamma_{h h} G_{n x} .
$$


Equation (27) implies that the first summand is nonnegative. Next, we show that $G_{n x}$ is strictly positive, which allows us to conclude that $\tilde{n}^{T}(x)$ is strictly increasing in $x$. Using the definition of $\Omega$, we have

$$
G_{n x}=\Omega^{-2}\left(f^{\prime} h g_{U I}+\left(f-f^{\prime} n h\right) \cdot\left[g_{U I}-\bar{h} T \cdot g_{S T C}+\left(T \cdot g_{S T C}+\tau\right) h\right]\right) .
$$

This is strictly positive as $g_{U I}-\bar{h} T \cdot g_{S T C} \geq 0$, and since strict concavity of $f$ ensures $f-f^{\prime} n h>0$.

So far we have shown that $\tilde{n}^{T}(x)$ is strictly increasing in $x$, and that $\tilde{h}^{T}(x)$ is weakly decreasing in $x$, strictly so if $g_{U I}-\bar{h} T \cdot g_{S T C}>0$. Next, we derive the result mentioned in Footnote 18: the marginal utility of consumption, which is equal to the Lagrange multiplier for the budget constraint, is strictly decreasing in $x$ in the relaxed problem. Let $\tilde{\lambda}^{T}(x)$ denote its value at the optimal solution. For the case $g_{U I}-\bar{h} T \cdot g_{S T C}>0$, Proposition 1 establishes a strictly increasing relationship between $\tilde{\lambda}^{T}(x)$ and $\tilde{h}^{T}(x)$. Since $\tilde{h}^{T}(x)$ is strictly decreasing, so is $\tilde{\lambda}^{T}(x)$. Next, consider the case $g_{U I}-\bar{h} T \cdot g_{S T C}=0$. Equation (23) implies $\log (\lambda)=\sigma[\log (\Gamma)-\log (\Omega)]$. Since hours are independent of $x$ in this case, we have

$$
\frac{d}{d x} \log \left(\tilde{\lambda}^{T}(x)\right)=\sigma\left[\frac{\Gamma_{n}}{\Gamma}-\frac{\Omega_{n}}{\Omega}\right] \frac{d}{d x} \tilde{n}^{T}(x)-\sigma \frac{\Omega_{x}}{\Omega}=-\sigma \frac{1-\psi}{\psi} \frac{\Gamma_{n}}{\Gamma} \frac{d}{d x} \tilde{n}^{T}(x)-\sigma \frac{\Omega_{x}}{\Omega},
$$

where the second equality uses the first-order condition $G_{n}=0$. This expression is strictly negative, since $\Omega_{x}>0, \psi \in(0,1), \Gamma_{n}>0$, and $\frac{d}{d x} \tilde{n}^{T}(x)>0$.

Since $\tilde{n}^{T}(x)$ is strictly increasing, we can determine the unique threshold $x_{N}^{T}$ at which (5) becomes binding. If $\lim _{x \rightarrow 0} n^{T}(x) \geq 1$, let $x_{N}^{T}=0$. If $\lim _{x \rightarrow \infty} n^{T}(x) \leq 1$, let $x_{N}^{T}=+\infty$. Otherwise, let $x_{N}^{T}$ be the unique level of $x$ that satisfies $\tilde{n}^{T}(x)=1$.

So far we have studied the relaxed problem without constraints (5) and (6). Next, we modify this problem by imposing (5) with equality. Let $\tilde{h}_{N}^{T}(x)$ denote the level of hours that maximizes $G(1, h, x)$. It is strictly increasing if $G_{h x}>0$. Evaluated at $n=1$, the sign of $G_{h x}$ equals the sign of

$$
f^{\prime} \Omega-\Omega_{h} f=f^{\prime}\left[x f-\tau h+(\bar{h}-h) g_{S T C}\right]-\left[x f^{\prime}-\tau-g_{S T C}\right] f=f^{\prime} \bar{h} g_{S T C}+\left[f-f^{\prime} h\right] \cdot\left[\tau+g_{S T C}\right],
$$

where the first equality substitutes $\Omega$ and $\Omega_{h}$, and the second equality exploits cancelations. The resulting expression is strictly positive, thus $\tilde{h}_{N}^{T}(x)$ is strictly increasing in $x$.

As the final preliminary step, we need to determine how employment varies when (6) is binding. Let $\hat{n}^{T}(x, h)$ denote the level of employment that maximizes $G(n, h, x)$ for a given level of hours $h$. Above we established that $G_{n x}>0$, hence $\hat{n}^{T}(x, h)$ is strictly increasing in $x$.

Using these preliminary results, we are ready prove the first two parts of the proposition.

1. Across levels of $x$ with a slack (5), hours and employment are $h^{0}(x)=\tilde{h}^{0}(x)$ and $n^{0}(x)=\tilde{n}^{0}(x)$, 
respectively. Constraint (5) becomes binding at $x_{N}^{0}$. Hours $h^{0}(x)$ are strictly decreasing since the condition $g_{U I}-\bar{h} T \cdot g_{S T C}>0$ is satisfied for $T=0$, as $g_{U I}>0$. Employment $n^{0}(x)$ is strictly increasing on $\left(0, x_{N}^{0}\right)$. On $\left(x_{N}^{0},+\infty\right)$, employment $n^{0}(x)$ equals one. Hours are $h^{0}(x)=\tilde{h}_{N}^{0}(x)$ on this interval and thus strictly increasing.

2. First, we determine $x_{M H R, L}^{1}$. Consider the decreasing function $\tilde{h}^{1}(x)$. If $\lim _{x \rightarrow x_{N}^{1}} \tilde{h}^{1}(x) \geq g_{M H R} \bar{h}$, set $x_{M H R, L}^{1}=x_{N}^{1}$. If $\lim _{x \rightarrow 0} \tilde{h}^{1}(x) \leq g_{M H R} \bar{h}$, set $x_{M H R, L}^{1}=0$. Otherwise, let $x_{M H R, L}^{1}$ be the unique level of $x \in\left(0, x_{N}^{1}\right)$ that satisfies $\tilde{h}^{1}(x)=g_{M H R} \bar{h}$. Next, we determine $x_{M H R, H}^{1}$. Consider the strictly increasing function $\tilde{h}_{N}^{1}(x)$. If $\lim _{x \rightarrow x_{N}^{1}} \tilde{h}_{N}^{1}(x) \geq g_{M H R} \bar{h}$, let $x_{M H R, H}^{1}=x_{N}^{1}$. If $\lim _{x \rightarrow \infty} \tilde{h}_{N}^{1}(x) \leq g_{M H R} \bar{h}$, let $x_{M H R, H}^{1}=\infty$. Otherwise, let $x_{M H R, H}^{1}$ be the unique level of $x \in\left(x_{N}^{1}, \infty\right)$ that satisfies $\tilde{h}_{N}^{1}(x)=g_{M H R} \bar{h}$. Having constructed these thresholds, we have

$$
\left(h^{1}(x), n^{1}(x)\right)= \begin{cases}\left(g_{M H R} \bar{h}, \hat{n}^{1}\left(x, g_{M H R} \bar{h}\right)\right) & \text { for } x \in\left(0, x_{M H R, L}^{1}\right), \\ \left(\tilde{h}^{1}(x), \tilde{n}^{1}(x)\right) & \text { for } x \in\left(x_{M H R, L}^{1}, x_{N}^{1}\right), \\ \left(\tilde{h}_{N}^{1}(x), 1\right) & \text { for } x \in\left(x_{N}^{1}, x_{M H R, H}^{1}\right), \\ \left(g_{M H R} \bar{h}, 1\right) & \text { for } x \in\left(x_{M H R, H}^{1},+\infty\right) .\end{cases}
$$

The properties of the functions $\hat{n}^{1}\left(x, g_{M H R} \bar{h}\right), \tilde{h}^{1}(x), \tilde{n}^{1}(x)$, and $\tilde{h}_{N}^{1}(x)$ imply that $h^{1}(x)$ and $n^{1}(x)$ vary with $x$ as stated in the proposition.

For Part 3 of the proposition, we first establish two preliminary results, again starting with the solution to the relaxed problem, that is, the functions $\tilde{h}^{T}(x)$ and $\tilde{n}^{T}(x)$. So far, we have defined these functions for $T \in\{0,1\}$. We now extend this definition to $T \in[0,1]$, letting take-up vary continuously. Using $\Omega_{T}=n(\bar{h}-h) g_{S T C}, \Omega_{n T}=(\bar{h}-h) g_{S T C}$, and $\Omega_{h T}=-n g_{S T C}$, second derivatives involving take-up are

$$
G_{n T}=\Omega^{-2}\left(\Omega-\Omega_{n} n\right)(\bar{h}-h) g_{S T C} \quad \text { and } \quad G_{h T}=-\Omega^{-2}\left(\Omega+\Omega_{h}(\bar{h}-h)\right) n g_{S T C} .
$$

Hours $\tilde{h}^{T}(x)$ are strictly decreasing in $T$ if $-G_{n n} G_{h T}+G_{n h} G_{n T}$ is strictly negative. Substituting the second derivatives into this expression, dropping $\Omega^{2}$, and exploiting cancelations, we obtain

$$
-g_{S T C}\left\{x\left|f^{\prime \prime}\right| h n \Omega\left[(\bar{h}-h)\left(\Omega_{h} h-\Omega_{n} n\right)+\Omega \bar{h}\right]+(1-\psi) \Omega \Omega_{n}\left[\Omega_{n} n+\Omega_{h}(\bar{h}-h)\right]\right\} .
$$

Equation (27) implies $h \Omega_{h}-n \Omega_{n} \geq 0$. Thus the preceding expression is strictly negative if $h \leq \bar{h}$. If $\tilde{h}^{0}(x) \leq \bar{h}, \tilde{h}^{T}(x)$ is then strictly decreasing in $T$ at $T=0$. This ensures that $\tilde{h}^{T}(x)$ remains below $\bar{h}$ as $T$ increases towards one. This yields a first preliminary result: $\tilde{h}^{0}(x) \leq \bar{h}$ implies $\tilde{h}^{1}(x)<\tilde{h}^{0}(x)$. 
Next, we modify the relaxed problem by imposing (5) with equality. The optimal level of hours in this problem is $\tilde{h}_{N}^{T}(x)$, now defined for $T \in[0,1]$. Hours $\tilde{h}_{N}^{T}(x)$ are strictly decreasing in $T$ if $G_{h T}<0$. If $\tilde{h}_{N}^{0}(x) \leq \bar{h}$, then $G_{h T}<0$ is satisfied at $T=0$. Thus $\tilde{h}_{N}^{T}(x)$ remains below $\bar{h}$ as $T$ increases towards one. This yields a second preliminary result: $\tilde{h}_{N}^{0}(x) \leq \bar{h}$ implies $\tilde{h}_{N}^{1}(x)<\tilde{h}_{N}^{0}(x)$.

Using these preliminary results, we can prove Part 3 of the proposition:

3. If $h^{0}(x)>g_{M H R} \bar{h}$, then the result follows immediately from the MHR, which implies $h^{1}(x) \leq$ $g_{M H R} \bar{h}$. So suppose $h^{0}(x) \leq g_{M H R} \bar{h}$. There are four cases. First, suppose $x \leq \min \left[x_{N}^{0}, x_{N}^{1}\right]$. Then $h^{0}(x)=\tilde{h}^{0}(x)$. Since $\tilde{h}^{0}(x) \leq g_{M H R} \bar{h} \leq \bar{h}$, the first preliminary result implies $\tilde{h}^{1}(x)<\tilde{h}^{0}(x)$. Thus the MHR does not bind, and $h^{1}(x)=\tilde{h}^{1}(x)<h^{0}(x)$. Second, consider $x \geq \max \left[x_{N}^{0}, x_{N}^{1}\right]$. Then $h^{0}(x)=\tilde{h}_{N}^{0}(x)$. Since $h_{N}^{0}(x) \leq g_{M H R} \bar{h} \leq \bar{h}$, the second preliminary result implies $\tilde{h}_{N}^{1}(x)<$ $\tilde{h}_{N}^{0}(x)$. Thus the MHR does not bind, and $h^{1}(x)=\tilde{h}_{N}^{1}(x)<h^{0}(x)$. Third, suppose that $x_{N}^{1}<$ $x_{N}^{0}$ and consider $x \in\left[x_{N}^{1}, x_{N}^{0}\right]$. Then $h^{0}(x)=\tilde{h}^{0}(x)$. Since $\tilde{h}^{0}$ is decreasing, we have $\tilde{h}^{0}(x) \geq$ $\tilde{h}^{0}\left(x_{N}^{0}\right)=\tilde{h}_{N}^{0}\left(x_{N}^{0}\right)$. Thus $\tilde{h}_{N}^{0}\left(x_{N}^{0}\right) \leq g_{M H R} \bar{h} \leq \bar{h}$, hence the second preliminary result ensures $\tilde{h}_{N}^{0}\left(x_{N}^{0}\right)>\tilde{h}_{N}^{1}\left(x_{N}^{0}\right)$. Since $\tilde{h}_{N}^{1}$ is increasing, it follows that $\tilde{h}_{N}^{1}\left(x_{N}^{0}\right) \geq \tilde{h}_{N}^{1}(x)$. Thus the MHR does not bind at $x$, and $h^{1}(x)=\tilde{h}_{N}^{1}(x)<h^{0}(x)$. Fourth, suppose that $x_{N}^{0}<x_{N}^{1}$ and consider $x \in\left[x_{N}^{0}, x_{N}^{1}\right]$. Here $h^{0}(x)=\tilde{h}_{N}^{0}(x)$. Since the function $\tilde{h}_{N}^{0}$ is strictly increasing, it follows that $\tilde{h}_{N}^{0}(x) \geq \tilde{h}_{N}^{0}\left(x_{N}^{0}\right)=\tilde{h}^{0}\left(x_{N}^{0}\right)$. This implies that $\tilde{h}^{0}\left(x_{N}^{0}\right) \leq g_{M H R} \bar{h} \leq \bar{h}$, hence the first preliminary result implies $\tilde{h}^{0}\left(x_{N}^{0}\right)>\tilde{h}^{1}\left(x_{N}^{0}\right)$. Since the function $\tilde{h}^{1}$ is decreasing, it follows that $\tilde{h}^{1}\left(x_{N}^{0}\right) \geq \tilde{h}^{1}(x)$. Thus the MHR does not bind at $x$, and $h^{1}(x)=\tilde{h}^{1}(x)<h^{0}(x)$.

\section{Proposition 5}

As a first step, we examine how the maximized value of the objective conditional on take-up varies with $x$. For given $x$ and $T$, the optimal value of the tuple $\left(c_{w}(x), c_{b}(x), n(x), h(x)\right)$ must maximize

$$
\begin{aligned}
& n(x) u\left(c_{w}(x), h(x)\right)+(1-n(x)) u\left(c_{b}(x), 0\right)+\lambda^{*}\{x f(n(x) h(x))-\tau n(x) h(x) \\
& \left.+(1-n(x)) g_{U I}+n(x)(\bar{h}-h(x)) T \cdot g_{S T C}-n(x) c_{w}(x)-(1-n(x)) c_{b}(x)\right\}
\end{aligned}
$$

subject to the constraints (5) and (6). The corresponding optimal values of labor inputs are $h^{T}(x)$ and $n^{T}(x)$. Let $U^{T}(x)$ denote the associated maximized value of objective (28). The optimal take-up decision $T^{*}(x)$ must maximize $U^{T}(x)$. By the envelope theorem, we have

$$
\frac{d U^{T}}{d x}(x)=\lambda^{*} f\left(n^{T}(x) h^{T}(x)\right) .
$$


Using this preliminary result, we now prove the proposition by sequentially analyzing the three regions $\left(0, x_{N}^{1}\right],\left[x_{N}^{1}, x_{N}^{0}\right]$, and $\left[x_{N}^{0},+\infty\right)$, thereby determining the location of the threshold $x_{T}$.

First, consider the interval $\left(0, x_{N}^{1}\right]$. According to Proposition 3, both $h^{0}(x)$ and $h^{1}(x)$ are constant over this interval. Furthermore, for both $T=0$ and $T=1$, employment is obtained by substituting hours into equation (22). Using the latter relationship to solve for $n^{T}(x) h^{T}(x)$ yields

$$
n^{T}(x) h^{T}(x)=\left(f^{\prime}\right)^{-1}\left(A^{T} / x\right)=\left(\alpha x / A^{T}\right)^{\frac{1}{1-\alpha}}
$$

where $A^{T}$ is a constant that depends on take-up $T$, and the second equality uses the assumption $f(n h)=$ $(n h)^{\alpha}$, which implies $\left(f^{\prime}\right)^{-1}(y)=(\alpha / y)^{1 /(1-\alpha)}$. Consequently, using equation (29) we obtain

$$
\frac{d}{d x}\left[U^{1}(x)-U^{0}(x)\right]=\lambda^{*}\left(\alpha x / A^{1}\right)^{\frac{\alpha}{1-\alpha}}\left[1-\left(A^{1} / A^{0}\right)^{\frac{\alpha}{1-\alpha}}\right]
$$

The sign of the RHS does not depend on $x$. Furthermore, $U^{T}(0)=u\left(c_{b}\left(\lambda^{*}\right), 0\right)+\lambda^{*}\left[g_{U I}-c_{b}\left(\lambda^{*}\right)\right]$ is independent of $T$. Consequently, if $A^{1}>A^{0}$, then $U^{1}(x)<U^{0}(x)$ for all $x \in\left(0, x_{N}^{1}\right]$. We set $x_{T}=0$ in this case. Below we show that no take-up is optimal at all levels of $x$ in this case. If $A^{1}<A^{0}$, then $U^{1}(x)>U^{0}(x)$ on $\left(0, x_{N}^{1}\right]$, and the threshold $x_{T}$ will lie to the right of this interval.

The case $A^{1}=A^{0}$ can also arise as part of the optimal solution. Given $\lambda^{*}$, the firm is then indifferent concerning take-up at any given $x \in\left(0, x_{N}^{1}\right]$. Meanwhile, the budget constraint requires that, across levels of $x$, a specific expected value of employment must be allocated to take-up. One optimal choice is to choose a threshold $x_{T} \in\left[0, x_{N}^{1}\right]$ and set $T^{*}(x)=1$ on $\left(0, x^{T}\right]$ and $T^{*}(x)=0$ on $\left(x^{T}, x_{N}^{1}\right]$. We proceed with this choice, and the precise value of $x_{T}$ is then determined by the budget constraint.

Next, consider the interval $\left[x_{N}^{1}, x_{N}^{0}\right]$. Here there are two cases, depending on the results for the interval $\left(0, x_{N}^{1}\right]$. First, if $U^{1}(x) \leq U^{0}(x)$ on $\left(0, x_{N}^{1}\right]$, then take-up remains inferior on $\left[x_{N}^{1}, x_{N}^{0}\right]$ : if (5) were not binding for take-up, then the analysis for the interval $\left(0, x_{N}^{1}\right]$ would directly extend to $\left[x_{N}^{1}, x_{N}^{0}\right]$, and the binding (5) makes take-up even less attractive. Second, there are two possibilities if $U^{1}(x)>U^{0}(x)$. Take-up may remain optimal on the entire interval $\left[x_{N}^{1}, x_{N}^{0}\right]$, or no take-up may be optimal on part of this interval. Next, we show that if there is a switch to no take-up somewhere within this interval, then no take-up remains optimal after this switch. Suppose there is a level $\tilde{x}_{T} \in\left[x_{N}^{1}, x_{N}^{0}\right]$ such that $U^{1}\left(\tilde{x}_{T}\right)=U^{0}\left(\tilde{x}_{T}\right)$. Using equation (29) and the fact that $n^{1}(x)=1$ on $\left[x_{N}^{1}, x_{N}^{0}\right]$, we have

$$
\frac{d}{d x}\left[U^{1}(x)-U^{0}(x)\right]=\lambda^{*}\left[f\left(h^{1}(x)\right)-f\left(n^{0}(x) h^{0}(x)\right)\right] .
$$

Since $U^{1}(x)>U^{0}(x)$ on $\left(0, x_{N}^{1}\right]$, this derivative must be strictly positive at $x=x_{N}^{1}$. To permit $U^{1}\left(\tilde{x}_{T}\right)=$ 
$U^{0}\left(\tilde{x}_{T}\right)$, this derivative must turn strictly negative somewhere between $x_{N}^{1}$ and $\tilde{x}_{T}$. Consequently, there must exist a level $x_{D} \in\left[x_{N}^{1}, \tilde{x}_{T}\right]$ such that

$$
h^{1}\left(x_{D}\right)<n^{0}\left(x_{D}\right) h^{0}\left(x_{D}\right) .
$$

Now consider $x \in\left[x_{D}, x_{N}^{0}\right]$. Equation (30) implies

$$
\left(x / x_{D}\right)^{\frac{1}{1-\alpha}} n^{0}\left(x_{D}\right) h^{0}\left(x_{D}\right)=n^{0}(x) h^{0}(x) .
$$

Using the notation from the proof of Proposition 3, let $\tilde{h}_{N}^{T}\left(x, \lambda^{*}\right)$ denote the solution to equation (19). Using the assumption $f(h)=h^{\alpha}$, equation (19) can be written as

$$
h=(\alpha x)^{\frac{1}{1-\alpha}}\left[\left(\lambda^{*}\right)^{-\frac{1}{\sigma}} V(h)+\tau+T \cdot g_{S T C}\right]^{-\frac{1}{1-\alpha}} .
$$

Thus

$$
\tilde{h}_{N}^{1}\left(x, \lambda^{*}\right)=\left[\left(\frac{x}{x_{D}}\right) \frac{\left(\lambda^{*}\right)^{-\frac{1}{\sigma}} V\left(\tilde{h}_{N}^{1}\left(x_{D}, \lambda^{*}\right)\right)+\left(\tau+g_{S T C}\right)}{\left(\lambda^{*}\right)^{-\frac{1}{\sigma}} V\left(\tilde{h}_{N}^{1}\left(x, \lambda^{*}\right)\right)+\left(\tau+g_{S T C}\right)}\right]^{\frac{1}{1-\alpha}} \tilde{h}_{N}^{1}\left(x_{D}, \lambda^{*}\right)<\left(\frac{x}{x_{D}}\right)^{\frac{1}{1-\alpha}} \tilde{h}_{N}^{1}\left(x_{D}, \lambda^{*}\right)
$$

where the inequality follows as $\tilde{h}_{N}^{1}\left(x, \lambda^{*}\right)$ is strictly increasing on $\left[x_{N}^{1}, x_{N}^{0}\right]$ and $V(h)$ is strictly increasing. As $h^{1}(x)=\min \left[\tilde{h}_{N}^{1}\left(x, \lambda^{*}\right), g_{M H R} \bar{h}\right]$ and $\tilde{h}_{N}^{1}\left(x, \lambda^{*}\right)$ is strictly increasing, this inequality also implies

$$
h^{1}(x)<\left(x / x_{D}\right)^{\frac{1}{1-\alpha}} h^{1}\left(x_{D}\right) .
$$

Combining inequalities (32) and (34) with equation (33) yields $h^{1}(x)<n^{0}(x) h^{0}(x)$ for all $x \in\left[x_{D}, x_{N}^{0}\right]$. Since $x_{D} \leq \tilde{x}_{T}$ and $U^{1}\left(\tilde{x}_{T}\right)=U^{0}\left(\tilde{x}_{T}\right)$, equation (31) implies $U^{1}(x)<U^{0}(x)$ for all $x \in\left[\tilde{x}_{T}, x_{N}^{0}\right]$. Thus there is at most one value $\tilde{x}_{T}$ in $\left[x_{N}^{1}, x_{N}^{0}\right]$ such that $U^{1}\left(\tilde{x}_{T}\right)=U^{0}\left(\tilde{x}_{T}\right)$. If it exists, we set the threshold $x_{T}$ to this value $\tilde{x}_{T}$. Otherwise take-up remains optimal throughout the interval $\left[x_{N}^{1}, x_{N}^{0}\right]$.

Finally, consider the interval $\left[x_{N}^{0},+\infty\right)$. Over this range

$$
\frac{d}{d x}\left[U^{1}(x)-U^{0}(x)\right]=\lambda^{*}\left[f\left(h^{1}(x)\right)-f\left(h^{0}(x)\right)\right] .
$$

This is strictly negative, as $h^{1}(x)<h^{0}(x)$ from Part 3 of Proposition 3. There are two cases, depending on the results obtained for the intervals $\left(0, x_{N}^{1}\right]$ and $\left[x_{N}^{1}, x_{N}^{0}\right]$. In the first, we have already set a threshold $x_{T} \in\left[0, x_{N}^{0}\right]$ such that $T^{*}(x)=0$ on $\left(x_{T}, x_{N}^{0}\right]$. Inequality (35) then implies that no take-up $T^{*}(x)=0$ is 
also optimal on $\left[x_{N}^{0},+\infty\right)$. In the second case, we have not yet determined a threshold $x_{T}$, and $T^{*}(x)=1$ is optimal on $\left(0, x_{N}^{0}\right]$. Inequality (35) then implies that there is at most one level $\tilde{x}_{T}$ in $\left[x_{N}^{0},+\infty\right)$ such that $U^{1}\left(\tilde{x}_{T}\right)=U^{0}\left(\tilde{x}_{T}\right)$. If such a level exists, we set $x_{T}=\tilde{x}_{T}$. Otherwise we set $x_{T}=+\infty$. With this definition, $T^{*}(x)=1$ is optimal on $\left[x_{N}^{0}, x_{T}\right]$, and $T^{*}(x)=0$ is optimal on $\left(x_{T},+\infty\right)$.

\section{B Sensitivity Analysis}

In this appendix we show that the main conclusions obtained in the computational experiments of Section 4 are not sensitive with respect to changes in parameters and targets.

Recall that the parameters $\sigma, \alpha, v$, and $\sigma_{x}$ were chosen independently, while $v_{0}, g_{U I}$, and $\psi$ were pinned down by targets for temporary layoffs, the replacement rate of UI, and the Frisch elasticity. For each parameter in the first group we choose a low and a high value. Similarly, for each of the three targets we choose a low and a high value. We vary one parameter or target at a time, and for each deviation from the benchmark we recalibrate the model and repeat the welfare analysis.

Table 8 and Table 9 display the results for the cases of perfect PI and no PI, respectively. We omit the results for the production function parameter $\alpha$, since changing this parameter has very little impact on the welfare effects associated with STC. We also do not report results for the fraction of unattached workers, since it plays a role very similar to that of risk aversion $\sigma \cdot{ }^{33}$ For each change in one of the remaining parameters and targets, we present the results in a pair of rows. The first of these rows, labeled 'Policy', displays the values of the policy instruments for the respective policy experiment. The second row, labeled 'Welfare', displays welfare relative to the experiment $g_{U I}^{*}$ in consumption equivalents. The first pair of rows provides this information for the benchmark calibration.

\section{B.1 Perfect Private Insurance}

Our two main results of Section 4.2 are robust with respect to these changes in parameters and targets.

First, introducing STC can always improve on UI, with sizable welfare gains varying between $0.1 \%$ and $1.5 \%$ for experiment $g_{S T C}^{*} \mid g_{U I}^{*}$ and between $0.1 \%$ and $2.4 \%$ for experiment $\left(g_{S T C}, g_{U I}\right)^{*}$. When we exclude the experiments involving a change in the degree of risk aversion, these gains vary between $0.2 \%$ and $0.5 \%$, and between $0.5 \%$ and $0.8 \%$, respectively. Naturally, the degree of risk aversion is a key determinant of the magnitude of welfare gains. With high risk aversion, the motivation to insure

\footnotetext{
${ }^{33}$ The benefit of UI is to insure this group of workers, and the magnitude of this benefit is determined by risk aversion in conjunction with the size of this group.
} 
unattached workers is stronger, leading to a higher optimal level of $g_{U I}^{*}$. The composition of labor inputs is more distorted, leaving more room for STC to mitigate these distortions.

Second, optimal levels of STC are markedly less generous than UI, and the results for the experiment $\bar{g}_{S T C}^{\max } \mid g_{U I}^{*}$ show that introducing STC with the same generosity as $g_{U I}$ results in large welfare losses.

Another robust result is that the welfare gains from STC are about equally distributed between the direct gain of introducing STC for a given level of UI and the additional gain from jointly optimizing the levels of UI and STC.

Finally, the introduction of a minimum hours reduction does not generally lead to sizable welfare improvements. In most experiments, the gains are less than a third of welfare gains achievable by STC alone and negligible for some experiments.

\section{B.2 No Private Insurance}

As in the benchmark calibration for the no-PI scenario, welfare gains from the introduction of STC are generally negligible. Small but non-negligible gains arise for high risk aversion and a low targeted replacement rate. However, in both cases the welfare gains remain an order of magnitude below the gains obtained in the corresponding sensitivity analysis under perfect PI. 
Table 8: Sensitivity Analysis, Perfect PI

\begin{tabular}{llcccccc}
\hline \hline & & $g_{U I}^{*}$ & $g_{S T C}^{*} \mid g_{U I}^{*}$ & $g_{S T C}^{\text {max }} \mid g_{U I}^{*}$ & $\left(g_{U I}, g_{S T C}\right)^{*}$ & $\left(g_{S T C}, g_{M H R}\right)^{*} \mid g_{U I}^{*}$ & $\left(g_{U I}, g_{S T C}, g_{M H R}\right)^{*}$ \\
\hline Benchmark & Policy & 0.262 & $0.08 \mid 0.262$ & $0.308 \mid 0.262$ & $(0.284,0.13)$ & $(0.0643,0.809) \mid 0.262$ & $(0.28,0.0933,0.825)$ \\
& Welfare & 0 & 0.3011 & -1.858 & 0.5263 & 0.4027 & 0.6079 \\
\hline$\sigma=5$ & Policy & 0.295 & $0.166 \mid 0.295$ & $0.367 \mid 0.295$ & $(0.302,0.183)$ & $(0.166,0.916) \mid 0.295$ & $(0.302,0.183,0.905)$ \\
& Welfare & 0 & 1.527 & -0.3053 & 2.401 & 1.527 & 2.401 \\
\hline$\sigma=1$ & Policy & 0.24 & $0.0436 \mid 0.24$ & $0.27 \mid 0.24$ & $(0.255,0.0679)$ & $(0.0442,0.797) \mid 0.24$ & $(0.256,0.0589,0.807)$ \\
& Welfare & 0 & 0.07122 & -1.641 & 0.1153 & 0.1326 & 0.1983 \\
\hline$\sigma_{x}=0.15$ & Policy & 0.275 & $0.0882 \mid 0.275$ & $0.33 \mid 0.275$ & $(0.295,0.13)$ & $(0.0717,0.735) \mid 0.275$ & $(0.295,0.0966,0.752)$ \\
& Welfare & 0 & 0.3385 & -2.234 & 0.5255 & 0.4707 & 0.6686 \\
\hline$\sigma_{x}=0.05$ & Policy & 0.249 & $0.0629 \mid 0.249$ & $0.286 \mid 0.249$ & $(0.27,0.123)$ & $(0.0499,0.888) \mid 0.249$ & $(0.27,0.123,0.941)$ \\
& Welfare & 0 & 0.234 & -1.644 & 0.5531 & 0.2881 & 0.5531 \\
\hline Frisch Elasticity 1 & Policy & 0.26 & $0.0687 \mid 0.26$ & $0.319 \mid 0.26$ & $(0.287,0.127)$ & $(0.0523,0.776) \mid 0.26$ & $(0.281,0.084,0.801)$ \\
& Welfare & 0 & 0.3293 & -2.805 & 0.6634 & 0.4171 & 0.6836 \\
\hline Frisch Elasticity 0.4 & Policy & 0.266 & $0.0983 \mid 0.266$ & $0.293 \mid 0.266$ & $(0.279,0.135)$ & $(0.0872,0.86) \mid 0.266$ & $(0.279,0.11,0.867)$ \\
& Welfare & 0 & 0.2336 & -0.7572 & 0.3365 & 0.3422 & 0.4554 \\
\hline Temp. Layoffs 0.025 & Policy & 0.253 & $0.084 \mid 0.253$ & $0.295 \mid 0.253$ & $(0.275,0.136)$ & $(0.0663,0.814) \mid 0.253$ & $(0.271,0.0968,0.83)$ \\
& Welfare & 0 & 0.3336 & -1.55 & 0.5789 & 0.4307 & 0.644 \\
\hline Temp. Layoffs 0.005 05 & Policy & 0.279 & $0.0734 \mid 0.279$ & $0.331 \mid 0.279$ & $(0.299,0.121)$ & $(0.0612,0.8) \mid 0.279$ & $(0.297,0.0879,0.816)$ \\
& Welfare & 0 & 0.2508 & -2.446 & 0.4422 & 0.3582 & 0.5492 \\
\hline Rep. Rate 30\% & Policy & 0.3 & $0.0654 \mid 0.3$ & $0.367 \mid 0.3$ & $(0.319,0.109)$ & $(0.0579,0.79) \mid 0.3$ & $(0.319,0.0821,0.805)$ \\
& Welfare & 0 & 0.1955 & -3.429 & 0.3466 & 0.307 & 0.4797 \\
\hline Rep. Rate 20\% & Policy & 0.227 & $0.0986 \mid 0.227$ & $0.259 \mid 0.227$ & $(0.25,0.154)$ & $(0.0749,0.833) \mid 0.227$ & $(0.245,0.111,0.851)$ \\
& Welfare & 0 & 0.4621 & -0.677 & 0.7715 & 0.5367 & 0.773 \\
\hline \hline
\end{tabular}


Table 9: Sensitivity Analysis: No PI

\begin{tabular}{|c|c|c|c|c|c|c|}
\hline & & $g_{U I}^{*}$ & $\overline{g_{S T C}^{*} \mid g_{U I}^{*}}$ & $\left(g_{U I}, g_{S T C}\right)^{*}$ & $\left(g_{S T C}, g_{M H R}\right)^{*} \mid g_{U I}^{*}$ & $\left(g_{U I}, g_{S T C}, g_{M H R}\right)^{*}$ \\
\hline \multirow[t]{2}{*}{ Benchmark } & Policy & 0.25 & $0.00316 \mid 0.25$ & $(0.25,0.00331)$ & $(0.00314,0.996) \mid 0.25$ & $(0.25,0.00329,0.996)$ \\
\hline & Welfare & 0 & 0.0009286 & 0.0009721 & 0.0009316 & 0.0009749 \\
\hline \multirow[t]{2}{*}{$\sigma=5$} & Policy & 0.271 & $0.0541 \mid 0.271$ & $(0.276,0.0796)$ & $(0.0541,0.984) \mid 0.271$ & $(0.276,0.0796,0.976)$ \\
\hline & Welfare & 0 & 0.2711 & 0.4612 & 0.2711 & 0.4612 \\
\hline \multirow[t]{2}{*}{$\sigma=1$} & Policy & 0.236 & $0 \mid 0.236$ & $(0.236,0)$ & $(0,1) \mid 0.236$ & $(0.236,0,1)$ \\
\hline & Welfare & 0 & -0 & $-1.249 e-012$ & -0 & $-1.222 e-012$ \\
\hline \multirow[t]{2}{*}{$\sigma_{x}=0.15$} & Policy & 0.258 & $0.000398 \mid 0.258$ & $(0.258,0.000406)$ & 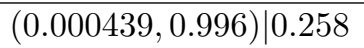 & $(0.258,0.000439,0.996)$ \\
\hline & Welfare & 0 & $1.072 e-005$ & $1.096 e-005$ & $1.562 e-005$ & $1.59 e-005$ \\
\hline \multirow[t]{2}{*}{$\sigma_{x}=0.05$} & Policy & 0.243 & $0.00338 \mid 0.243$ & $(0.244,0.00371)$ & $(0.00338,0.998) \mid 0.243$ & $(0.244,0.00371,0.998)$ \\
\hline & Welfare & 0 & 0.001878 & 0.002057 & 0.001878 & 0.002061 \\
\hline \multirow[t]{2}{*}{ Frisch Elasticity 1} & Policy & 0.249 & $0.00246 \mid 0.249$ & $(0.249,0.00258)$ & $(0.00244,0.996) \mid 0.249$ & $(0.249,0.00256,0.996)$ \\
\hline & Welfare & 0 & 0.0007345 & 0.0007715 & 0.0007384 & 0.0007751 \\
\hline \multirow[t]{2}{*}{ Frisch Elasticity 0.4} & Policy & 0.25 & $0.00414 \mid 0.25$ & $(0.25,0.00428)$ & $(0.00412,0.998) \mid 0.25$ & $(0.25,0.00426,0.998)$ \\
\hline & Welfare & 0 & 0.000995 & 0.001033 & 0.0009969 & 0.001034 \\
\hline \multirow[t]{2}{*}{ Temp. Layoffs 0.025} & Policy & 0.243 & $0.0043 \mid 0.243$ & $(0.243,0.00455)$ & $(0.00427,0.996) \mid 0.243$ & $(0.243,0.00452,0.996)$ \\
\hline & Welfare & 0 & 0.001713 & 0.001812 & 0.001714 & 0.001813 \\
\hline \multirow[t]{2}{*}{ Temp. Layoffs 0.005} & Policy & 0.262 & $0.00133 \mid 0.262$ & $(0.262,0.00137)$ & $(0.00131,0.997) \mid 0.262$ & $(0.262,0.00136,0.997)$ \\
\hline & Welfare & 0 & 0.0001646 & 0.0001694 & 0.0001699 & 0.0001747 \\
\hline \multirow[t]{2}{*}{ Rep. Rate $30 \%$} & Policy & 0.293 & $0 \mid 0.293$ & $(0.293,0)$ & $(0,1) \mid 0.293$ & $(0.293,0,1)$ \\
\hline & Welfare & 0 & -0 & $-6.2 e-011$ & -0 & $-7.841 e-011$ \\
\hline \multirow[t]{2}{*}{ Rep. Rate $20 \%$} & Policy & 0.206 & $0.0153 \mid 0.206$ & $(0.208,0.0189)$ & $(0.0153,0.995) \mid 0.206$ & $(0.208,0.0189,0.994)$ \\
\hline & Welfare & 0 & 0.02069 & 0.02529 & 0.02069 & 0.02529 \\
\hline
\end{tabular}




\section{Redundancy of Experience Rating}

Redundancy of experience rating is a feature our model shares with those of BW and WH. It applies even to their models in which attached agents are heterogeneous in that some are workers while others are employers. It follows from perfect risk sharing among agents attached to a firm.

First, we establish redundancy for a version of our model in which both UI benefits and experience rating are allowed to differentiate between attached and unattached workers. Following BW and WH, experience rating imposes a tax on a firm which amounts to a fraction $e \in[0,1]$ of the total benefits received by the workers attached to that firm. We also allow for experience rating of unattached workers with factor $e^{\nu} \in[0,1] .{ }^{34}$ With experience rating, the net-transfer schedule for firms (2) becomes

$$
(1-e)\left[(1-n) g_{U I}+n \mathcal{I}\left[h \leq g_{M H R} \bar{h}\right] \cdot(\bar{h}-h) \cdot g_{S T C}\right]-\tau n h .
$$

The net benefit for unattached workers is $\left(1-e^{\nu}\right) g_{U I}^{\nu}$ with $g_{U I}^{\nu}$ denoting the corresponding gross benefit. Clearly this system is equivalent to an alternative system (distinguished by a check) given by $\check{g}_{U I}=$ $(1-e) g_{U I}, \check{g}_{S T C}=(1-e) g_{S T C}, \check{e}=0, \check{g}_{U I}^{\nu}=\left(1-e^{\nu}\right) g_{U I}^{\nu}$, and $\check{e}^{\nu}=0$.

In our model without experience rating, we restrict STC and UI to be uniform. As discussed in the text, this should be understood as a restriction on effective subsidies. Otherwise it has no content, as any differentiation can be implemented through experience rating. Next, we show that experience rating is redundant if the effective subsidy cannot differentiate between attached and unattached workers:

$$
(1-e) g_{U I}=\left(1-e^{\nu}\right) g_{U I}^{\nu}
$$

The system consisting of $\check{g}_{U I}, \check{g}_{S T C}$, and $\check{g}_{U I}^{\nu}$ and no experience rating continues to be equivalent. It satisfies $\check{g}_{U I}=\check{g}_{U I}^{\nu}$, so the UI benefit does not differentiate between attached and unattached workers.

\footnotetext{
${ }^{34} \mathrm{~A}$ natural level for this is zero, since these workers are not attached to a firm. However, one can also assume that these workers were previously attached to some firm, on whose owners the experience-rating tax is imposed. In our model of owner-operators, it is internally consistent to assume that unattached workers own the firm from which they became unattached. In their role as owners, they then pay the experience-rating tax induced by benefits they receive as workers.
} 


\section{References}

Arpaia, A., N. Curci, E. Meyermans, J. Peschner and F. Pierini, "Short-time working arrangements as response to cyclical fluctuations," European Economy Occasional Paper 64, European Commission, 2010.

Balleer, A., B. Gehrke, W. Lechthaler and C. Merkl, "Does short-time work save jobs? A business cycle analysis," European Economic Review 84 (2016), 99-122.

Blanchard, O. J. and J. Tirole, "The Joint Design of Unemployment Insurance and Employment Protection: A First Pass," Journal of the European Economic Association 6 (03 2008), 45-77.

Boeri, T. and H. Bruecker, "Short-Time Work Benefits Revisited: Some Lessons from the Great Recession," Economic Policy 26 (October 2011), 697-765.

Braun, H. And B. Brügemann, "Welfare Effects of Short-Time Compensation," Discussion paper, Tinbergen Institute, 2017.

Burdett, K. AND R. Wright, "Unemployment insurance and short-time compensation: The effects on layoffs, hours per worker, and wages," Journal of Political Economy (1989), 1479-1496.

Cahuc, P. And S. CARcillo, "Is short-time work a good method to keep unemployment down?" Nordic Economic Policy Review (2011), 133-164.

Chetтy, R., "Moral Hazard versus Liquidity and Optimal Unemployment Insurance," Journal of Political Economy 116 (2008), 173-234.

Comin, D. A. And T. Philippon, "The rise in firm-level volatility: Causes and consequences," in NBER Macroeconomics Annual 2005, Volume 20 (MIT Press, 2006), 167-228.

Cooper, R., M. Meyer and I. Schott, "The Employment and Productivity Effects of Short-Time Work in Germany," Working paper, 2016.

Costain, J. S. And M. Reiter, "Business cycles, unemployment insurance, and the calibration of matching models," Journal of Economic Dynamics and Control 32 (2008), 1120-1155.

Davis, S. J., J. Haltiwanger, R. Jarmin and J. Miranda, "Volatility and dispersion in business growth rates: Publicly traded versus privately held firms," in NBER Macroeconomics Annual 2006, Volume 21 (MIT Press, 2007), 107-180. 
Feldstein, M., "Temporary layoffs in the theory of unemployment," Journal of Political Economy (1976), 937-957.

FitzRoy, F. R. And R. A. HART, "Hours, Layoffs and Unemployment Insurance Funding: Theory and Practice in an International Perspective," Economic Journal 95 (September 1985), 700-713.

Fujita, S. And G. Moscarini, "Recall and Unemployment," Working Paper, December 2016.

HALL, R., "Reconciling cyclical movements in the marginal value of time and the marginal product of labor," Journal of Political Economy 117 (2009), 281-323.

Heathcote, J., K. Storesletten and G. L. Violante, "Quantitative Macroeconomics with Heterogeneous Households," Annual Review of Economics 1 (2009), 319-354.

HiJzen, A. And S. Martin, "The role of short-time work schemes during the global financial crisis and early recovery: a cross-country analysis," IZA Journal of Labor Policy 2 (December 2013), 1-31.

HiJzen, A. AND D. VEnn, "The Role of Short-Time Work Schemes during the 2008-09 Recession," OECD Social, Employment and Migration Working Papers 115, OECD Publishing, Jan 2011.

King, R. G., C. I. Plosser and S. T. Rebelo, "Production, growth and business cycles: I. The basic neoclassical model," Journal of Monetary Economics 21 (1988), 195-232.

Miyazaki, H. And H. M. NeARY, "Output, Work Hours and Employment in the Short Run of a Labour-managed Firm," Economic Journal 95 (December 1985), 1035-48.

OECD, "Employment Outlook," (2002).

Rosen, S., "Implicit Contracts: A Survey," Journal of Economic Literature 23 (September 1985), 114475.

Tilly, J. And K. Niedermayer, "Employment and Welfare Effects of Short-Time Work," Working paper, 2016.

Topel, R. H., "On layoffs and unemployment insurance," The American Economic Review 73 (1983), 541-559.

Van Audenrode, M. A., "Short-Time Compensation: Job Security, and Employment Contracts: Evidence from Selected OECD Countries.," Journal of Political Economy 102 (1994), 76 - 102.

WRight, R. AND J. HotchKISs, "A general model of unemployment insurance with or without shorttime compensation," Research in Labour Economics, (1988), 91-131. 